\title{
Link and knot invariants from non-abelian Yang-Baxter 2-cocycles
}

\author{
Marco A. Farinati* and Juliana García Galofre ${ }^{\dagger}$ \\ July 9,2015
}

\begin{abstract}
We define a knot/link invariant using set theoretical solutions $(X, \sigma)$ of the Yang-Baxter equation and non commutative 2-cocycles. We also define, for a given $(X, \sigma)$, a universal group $U_{n c}(X)$ governing all 2-cocycles in $X$, and we exhibit examples of computations.
\end{abstract}

\section{Introduction and preliminaries}

The first part of this work consist of a generalization to biquandles and the notion of non-commutative 2-cocycle given in $\mathrm{AG}$ for quandles. It is also a generalization to the non-commutative case of part of the work in [CEGS] for commutative cocycles. In this way, we obtain in principle new invariants for biquandles that do not come from quandles, admitting non-commutative 2-cocycles, that is, whose universal group (see section 2 or 3) is non abelian.

In the second section we define a universal group governing all 2-cocycles for a given biquandle $X$, that is, a group $U_{n c}(X)$ together with a 2-cocycle $\pi: X \times X \rightarrow U_{n c}(X)$ such that if $f: X \times X \rightarrow G$ is a non commutative 2-cocycles with values in a group $G$, then there is a unique group homomorphism $\widetilde{f}: U_{n c}(X) \rightarrow G$ such that $f=\widetilde{f} \pi$. For instance, if $U_{n c}(X)$ is the trivial group, then every 2-cocycle is trivial. On the opposite, if $U_{n c}(X)$ is nontrivial, this universal property says that it carries all information that any group could give using non commutative 2-cocycles.

In the third section, a reduced version of $U_{n c}$ is given, it depends on a map $\gamma: X \rightarrow$ $U_{n c}(X)$. The constructed group is called $U_{n c}^{\gamma}(X)$, in particular it is a group and there is given a 2-cocycle $\pi_{\gamma}: X \times X \rightarrow U_{n c}^{\gamma}(X)$ with the following property (Theorem 23): if $f: X \times X \rightarrow G$ is a 2-cocycle, then there exists a cohomologous (see definition 6) 2-cocycle $f_{\gamma}: X \times X \rightarrow G$ and a group homomorphism $\tilde{f}_{\gamma}$ such that $f_{\gamma}=\tilde{f}_{\gamma} \pi_{\gamma}$. Since the invariant defined in section 1 is unchanged for cohomologous cocycles (Proposition

${ }^{*}$ Member of CONICET. Partially supported by PIP $11220110100800 \mathrm{CO}$, and UBACYT 20021030100481BA, mfarinat@dm.uba.ar.

${ }^{\dagger}$ Partially supported by PIP $11220110100800 \mathrm{CO}$ and UBACYT 20021030100481BA, jgarciag@dm.uba.ar 
13), the invariant produced with $f$ is the same as the one coming from $f_{\gamma}$, so we see that all invariants are governed by the group $U_{n c}^{\gamma}$, which is, in general, smaller than $U_{n c}$.

In section 4 we exhibit some examples of computations. Most of them were performed using GAP2015]. We wish to heartily thank Leandro Vendramin for teaching us the basic facts on syntax and GAP programing, and helping us with our first (and not so firsts) steps implementing the algorithms we needed. An interesting observation on examples is that, if $(X, \sigma)$ is a solution of the Yang-Baxter equation, then also is $\bar{\sigma}:=\sigma^{-1}$, and if $\sigma$ makes $X$ into a biquandle (see definition below), then also $\bar{\sigma}$ gives a biquandle structure. One may suspect that $\bar{\sigma}$ is, in a sense, equivalent to $\sigma$ and probably gives no new information, but this is not the case: one may have $U_{n c}^{\gamma}(\sigma)=1$ (so that $\sigma$ gives always trivial invariants for any 2-cocycle $f$ ) but $\bar{\sigma}$ may give non trivial invariants. Section 5 end with concluding remarks.

Before going into section 1 we recall the notion of biquandle and quandle:

Definition 1. A set theoretical solution of the Yang-Baxter equation is a pair $(X, \sigma)$ where $\sigma: X \times X \rightarrow X \times X$ is a bijection satisfying

$$
(\mathrm{Id} \times \sigma)(\sigma \times \mathrm{Id})(\mathrm{Id} \times \sigma)=(\sigma \times \mathrm{Id})(\mathrm{Id} \times \sigma)(\sigma \times \mathrm{Id})
$$

Notation: $\sigma(x, y)=\left(\sigma^{1}(x, y), \sigma^{2}(x, y)\right)$ and $\sigma^{-1}(x, y)=\bar{\sigma}(x, y)$.

A solution $(X, \sigma)$ is called non-degenerated, or birack if in addition:

1. (left invertibility) for any $x, z \in X$ there exists a unique $y$ such that $\sigma^{1}(x, y)=z$,

2. (right invertibility) for any $y, t \in X$ there exists a unique $x$ such that $\sigma^{2}(x, y)=t$.

A birack is called biquandle if, given $x_{0} \in X$, there exists a unique $y_{0} \in X$ such that $\sigma\left(x_{0}, y_{0}\right)=\left(x_{0}, y_{0}\right)$. In other words, if there exists a bijective map $s: X \rightarrow X$ such that

$$
\{(x, y): \sigma(x, y)=(x, y)\}=\{(x, s(x)): x \in X\}
$$

Definition 2. A rack is a pair $(X, \triangleleft)$ where $\triangleleft: X \rightarrow X$ verifies

1. for all $x \in X$, the map $-\triangleleft x: X \rightarrow X(y \mapsto y \triangleleft x)$ is bijective, and

2. $(x \triangleleft y) \triangleleft z=(x \triangleleft z) \triangleleft(y \triangleleft z)$

A rack is called a quandle if $x \triangleleft x=x$ for all $x \in X$.

Biracks and biquandles generalizes racks and quandles respectively because a map $\sigma: X \times X \rightarrow X \times X$ of the form $\sigma(x, y)=(y, f(x, y))$ is a birack if and only if the operation $\triangleleft$ given by $x \triangleleft y:=f(x, y)$ is a rack, and $\sigma$ is a biquandle if and only if this rack is a quandle. In section 2 we review some examples of biquandles.

\section{Non-abelian 2-cocycles}

Let $(X, \sigma)$ be a biquandle and $\mathrm{H}$ a (not necessarily abelian) group.

Definition 3. A function $f: X \times X \rightarrow H$ is a braid non-commutative 2-cocycle if 
- $f\left(x_{1}, x_{2}\right) f\left(\sigma^{2}\left(x_{1}, x_{2}\right), x_{3}\right)=f\left(x_{1}, \sigma^{1}\left(x_{2}, x_{3}\right)\right) f\left(\sigma^{2}\left(x_{1}, \sigma^{1}\left(x_{2}, x_{3}\right)\right), \sigma^{2}\left(x_{2}, x_{3}\right)\right)$, and

- $f\left(\sigma^{1}\left(x_{1}, x_{2}\right), \sigma^{1}\left(\sigma^{2}\left(x_{1}, x_{2}\right), x_{3}\right)\right)=f\left(x_{2}, x_{3}\right)$

are satisfied for any $x_{1}, x_{2}, x_{3} \in X$.

Definition 4. If $f$ further satisfies $f(x, s(x))=1$ for all $x \in X$ then it is called of type $I$.

Remark 5. If $f$ is a braided non commutative 2-cocycle and $\lambda: X \rightarrow H$ is an arbitrary function, then

$$
f^{\prime}(x, y)=\lambda(x) f(x, y) \lambda^{-1}\left(\sigma^{2}(x, y)\right)
$$

is also a braided non commutative 2-coycle. If moreover $f$ is of type $\mathrm{I}$, and $\lambda(x)=\lambda(s(x))$ for all $x \in X$, then $f^{\prime}$ is also of type I.

Definition 6. Two cocycles $f, f^{\prime}$ are cohomologous $\left(f \sim f^{\prime}\right)$ if there is a function $\gamma: X \rightarrow H$ such that $\gamma(x)=\gamma(s(x))$ and

$$
f^{\prime}(x, y)=\gamma(x) f(x, y) \gamma^{-1}\left(\sigma^{2}(x, y)\right), \forall x, y \in X .
$$

Remark 7 . It is easy to see that $\sim$ is an equivalence relation.

An equivalence class is called a cohomology class. The set of cohomology classes is denoted by $H_{N C}^{2}(X, H)$. This definitions, in case $(X, \triangleleft)$ is a quandle and considering $\sigma(x, y)=(y, x \triangleleft y)$, agree with the ones in [CEGS], since in this case the second condition of definition 3 is trivial. As in the rack/quandle case, if $H$ is not commutative, $H_{N C}^{2}(X, H)$ need not to be a group, it is just a set.

Remark 8. If $H$ happens to be commutative and $f: X \times X \rightarrow H$ is a 2-cocycle in the non commutative sense, then $f$ is necessarily a (special type of) 2-cocycle with trivial coefficients in the sense of [CES], but our definition is more restrictive, because we ask for a set of equations of the form $a b=a^{\prime} b^{\prime}$ and $c=c^{\prime}$ (plus being type I), while in the usual abelian 2-cocycles the equation is of the form $a b c=a^{\prime} b^{\prime} c^{\prime}$ (plus being type I).

Remark 9. The first condition of definition 3 is invariant under inverting $\sigma$, namely, $f$ satisfies it for $\sigma$ if and only if $f$ does it for $\sigma^{-1}$. On the other hand, the second condition is not invariant under inverting $\sigma$. For example, if $(X, \triangleleft)$ is a rack and $\sigma(x, y)=(y, x \triangleleft y)$, then the second condition is trivially satisfied for any function $f$ (and hence, this definition is equivalent, in this setting, to the one given in [CEGS]), while for $\bar{\sigma}(x, y)=\left(y \triangleleft^{-1} x, x\right)$ means that $f$ must be invariant under the action of the Inner group associated to the $\operatorname{rack} X$.

\section{$1.1 \quad$ Weights}

Let $X$ be a biquandle, $H$ a group, $f: X \times X \rightarrow H$ a non-abelian 2-cocyle. Let $L=$ $K_{1} \cup \cdots \cup K_{r}$ be a classical oriented link diagram on the plane, where $K_{1}, \ldots, K_{r}$ are connected components, for some positive integer $r$. A coloring of $L$ by $X$ is a rule that assigns an element of $X$ to each semi-arc of $L$, in such a way that for every crossing
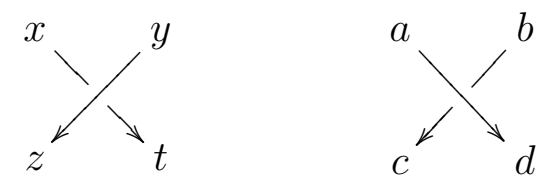
we have $(z, t)=\sigma(x, y)$ if the crossing is positive, and $(c, d)=\sigma^{-1}(a, b)$ if the crossing is negative.

Let $\mathcal{C} \in \operatorname{Col}_{X}(L)$ be a coloring of $L$ by $X$ and $\left(b_{1}, \ldots, b_{r}\right)$ a set of base points on the components $\left(K_{1}, \ldots, K_{r}\right)$. Let $\tau^{(i)}$, for $i=1, \ldots, r$ the set of crossings such that the under-arc is from the component $i$. Let $\left(\tau_{1}^{(i)}, \ldots, \tau_{k_{(i)}}^{(i)}\right)$ be the crossings in $\tau^{(i)}, i=1, \ldots, r$ such that appear in this order when one travels $K_{j}$ in the given orientation.

At a positive crossing $\tau$, let $x_{\tau}, y_{\tau}$ be the color on the incoming arcs. The Boltzmann weight at $\tau$ is $B_{f}(\tau, \mathcal{C})=f\left(x_{\tau}, y_{\tau}\right)$. At a negative crossing $\tau$, denote $\sigma\left(x_{\tau}, y_{\tau}\right)$ the colors on the incoming arcs. The Boltzmann weight at $\tau$ is $B_{f}(\tau, \mathcal{C})=f^{-1}\left(x_{\tau}, y_{\tau}\right)$

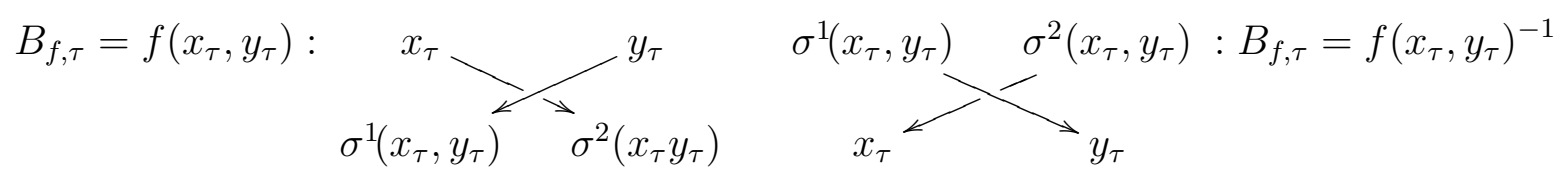

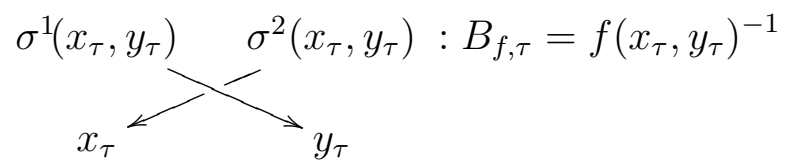

We will show that a convenient product of these weights is invariant under Reidemeister moves.

\section{$1.2 \quad$ Reidemeister type I moves}

First notice that $\sigma(x, s(x))=(x, s(x))$ implies $\sigma^{-1}(x, s(x))=(x, s(x))$, so, adding any orientation to the diagram
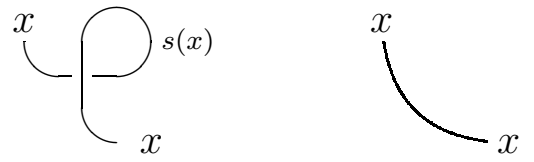

the condition $f^{ \pm 1}(x, s(x))=1$ assures that the factor due to this crossing do not count.

\subsection{Reidemeister type II moves}

We consider several cases:

Case 1:
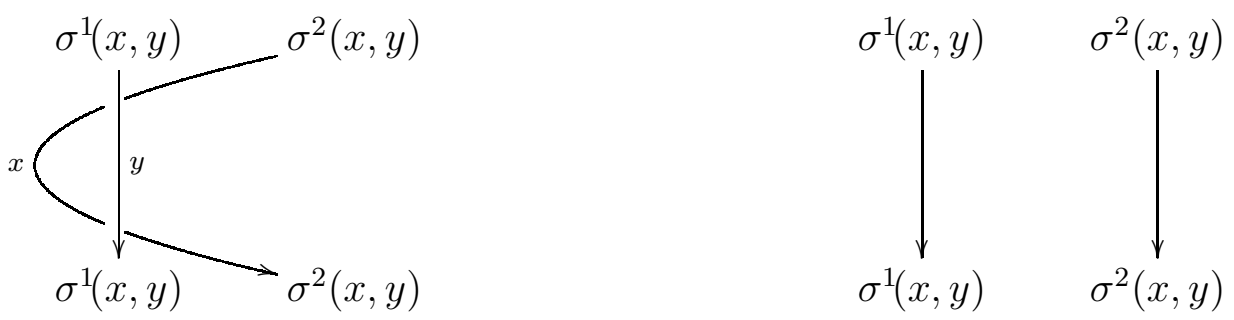

Case 2:
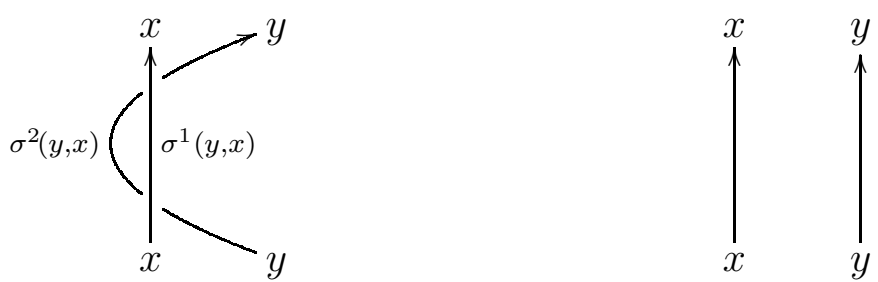
Case 3: In this case and the following, start naming the top arcs of the diagrams on the left, the rest of the arcs are known as $X$ is a biquandle.
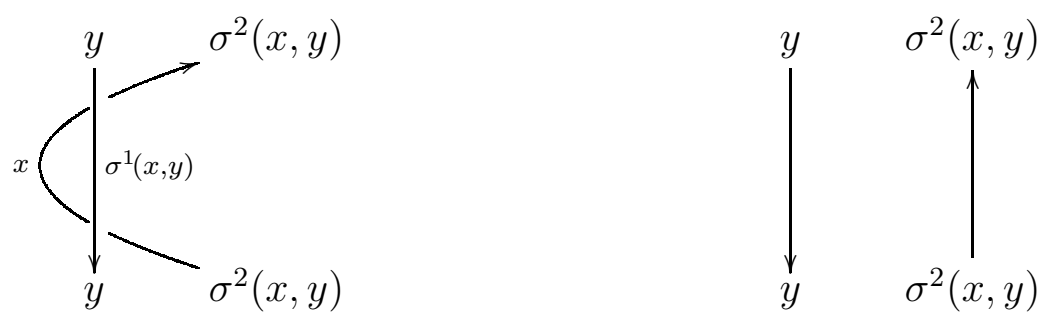

Case 4:
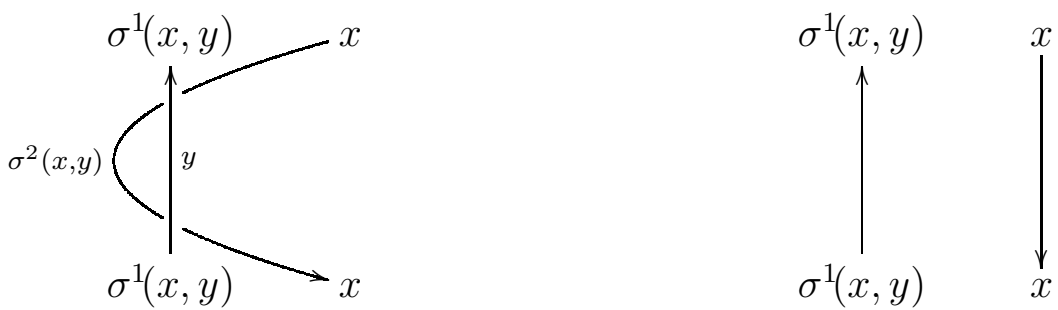

The product of weights corresponding to the diagrams on the left in cases 1 and 3 is $f^{-1}(x, y) f(x, y)=1$, in cases 2 and 4 is $f(x, y) f^{-1}(x, y)=1$.

\subsection{Reidemeister type III moves}

While there are eight oriented Reidemeister type III moves, only four of them are different.

Case 1: Start by naming the incoming-arcs $x_{1}, x_{2}, x_{3}$. In case 1 , as well as in the rest of the cases, once chosen three arcs in both diagrams the remaining arcs are respectively equal as $\sigma$ is a solution of YBeq.

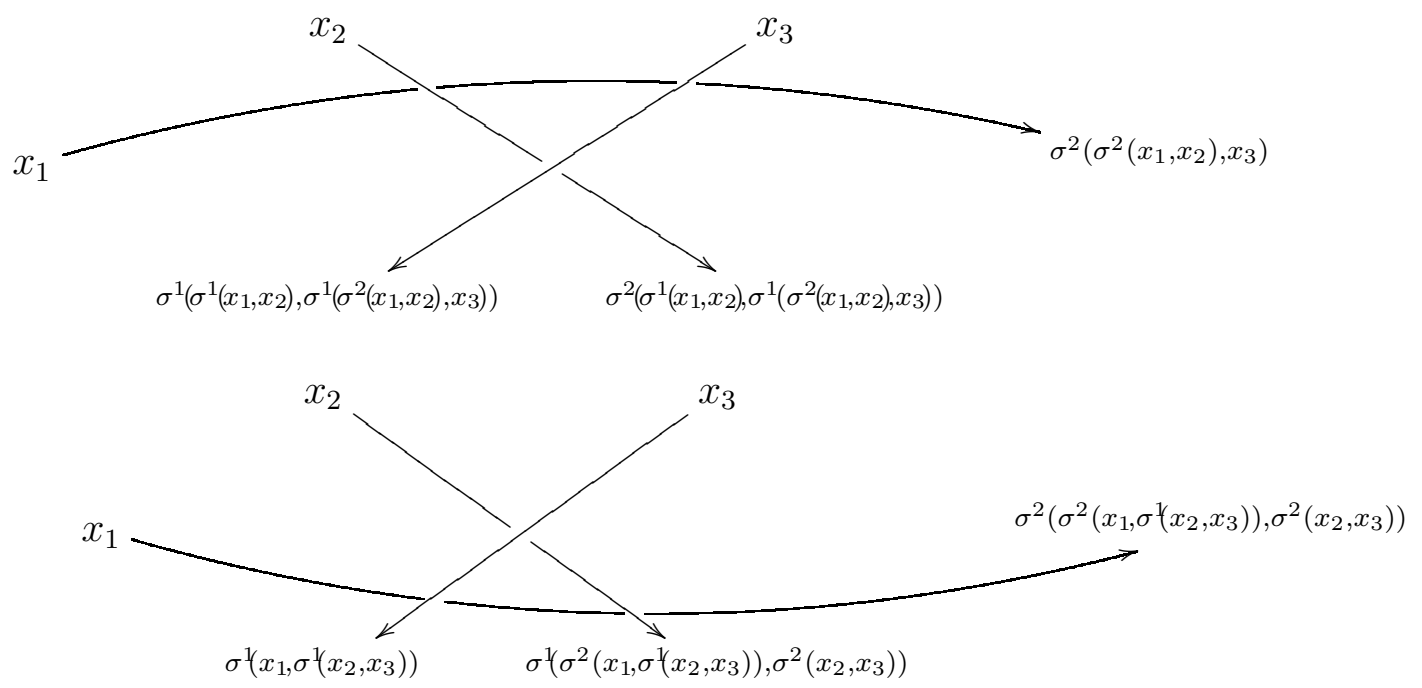

The product of the weights following the horizontal under-arc, in the first diagram, is:

$$
I=f\left(x_{1}, x_{2}\right) f\left(\sigma^{2}\left(x_{1}, x_{2}\right), x_{3}\right)
$$


and in the second, is:

$$
I I=f\left(x_{1}, \sigma^{1}\left(x_{2}, x_{3}\right)\right) f\left(\sigma^{2}\left(x_{1}, \sigma^{1}\left(x_{2}, x_{3}\right)\right), \sigma^{2}\left(x_{2}, x_{3}\right)\right)
$$

$I=I I$ is one of the equalities defining 2-cocycle, the other equation defining 2-cocycle affirms that the weights given to the other crossings are the same. Notice that in the quandle coloring this condition is trivial, but in the biquandle coloring it is not.

Case 2: Start by naming the $\operatorname{arcs} \sigma^{2}\left(x_{1}, x_{2}\right), x_{2}$ and $\sigma^{1}\left(x_{2}, x_{3}\right)$ in both diagrams. The remaining arcs are known using the fact that $X$ is a biquandle and $\sigma^{1}\left(\sigma^{1}\left(x_{1}, x_{2}\right), \sigma^{1}\left(\sigma^{2}\left(x_{1}, x_{2}\right), x_{3}\right)\right)=\sigma^{1}\left(x_{1}, \sigma^{1}\left(x_{2}, x_{3}\right)\right)$ (due to the braid equation).

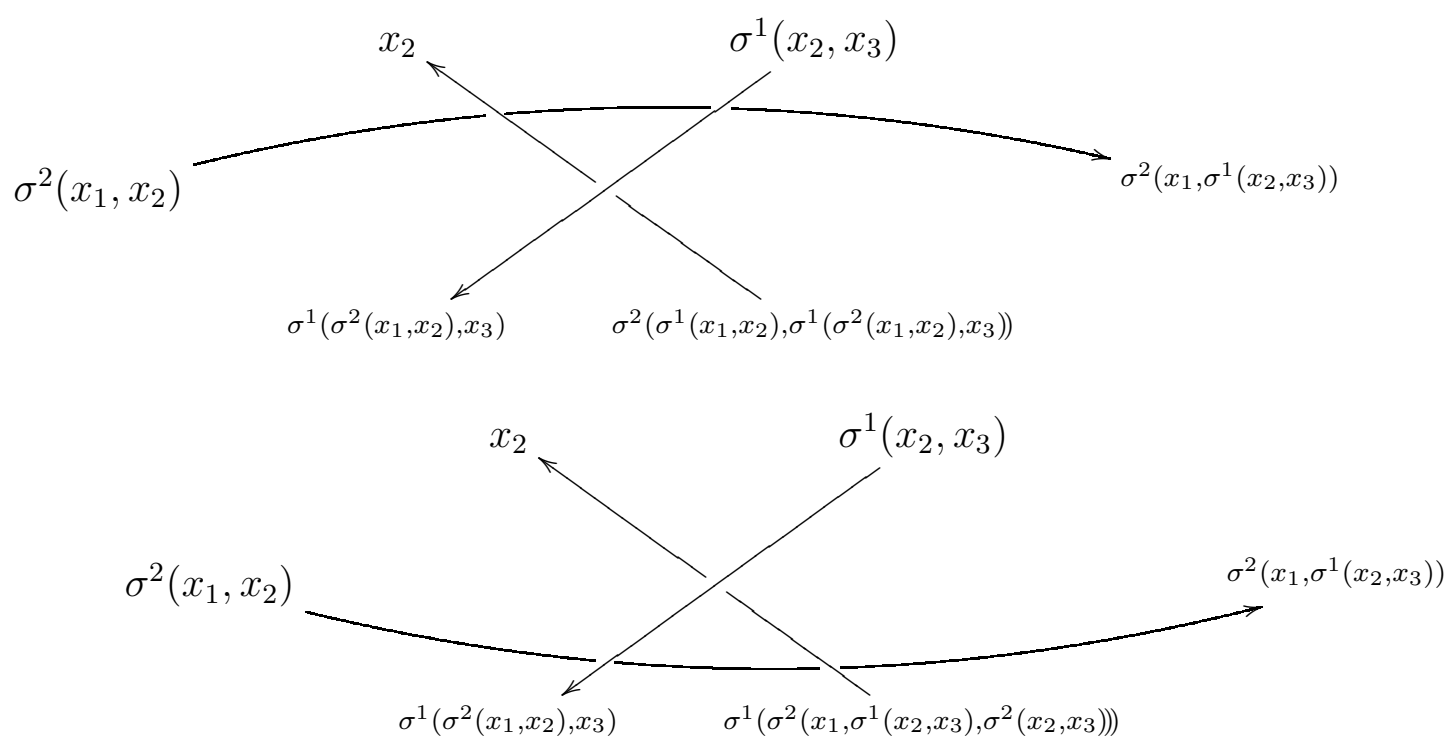

The product of weights for the horizontal line in the first diagram is

$$
I=f^{-1}\left(x_{1}, x_{2}\right) f\left(x_{1}, \sigma^{1}\left(x_{2}, x_{3}\right)\right)
$$

and for the second diagram is

$$
I I=f\left(\sigma^{2}\left(x_{1}, x_{2}\right), x_{3}\right) f^{-1}\left(\sigma^{2}\left(x_{1}, \sigma^{1}\left(x_{2}, x_{3}\right)\right), \sigma^{2}\left(x_{2}, x_{3}\right)\right)
$$

The remaining weights in both diagrams are $a=f^{-1}\left(\sigma^{1}\left(x_{1}, x_{2}\right), \sigma^{1}\left(\sigma^{2}\left(x_{1}, x_{2}\right), x_{3}\right)\right)$ and $b=f^{-1}\left(x_{2}, x_{3}\right)$. As $f$ is a 2-cocycle, $a=b$.

Case 3: Name the incoming arcs by $a, b$ and $c$.

Remark 10. YBeq is equivalent to the following equation, which explains the equality of the out-coming arcs in both diagrams.

$$
(\sigma \times 1)(1 \times \bar{\sigma})(\bar{\sigma} \times 1)=(1 \times \bar{\sigma})(\bar{\sigma} \times 1)(1 \times \sigma)
$$



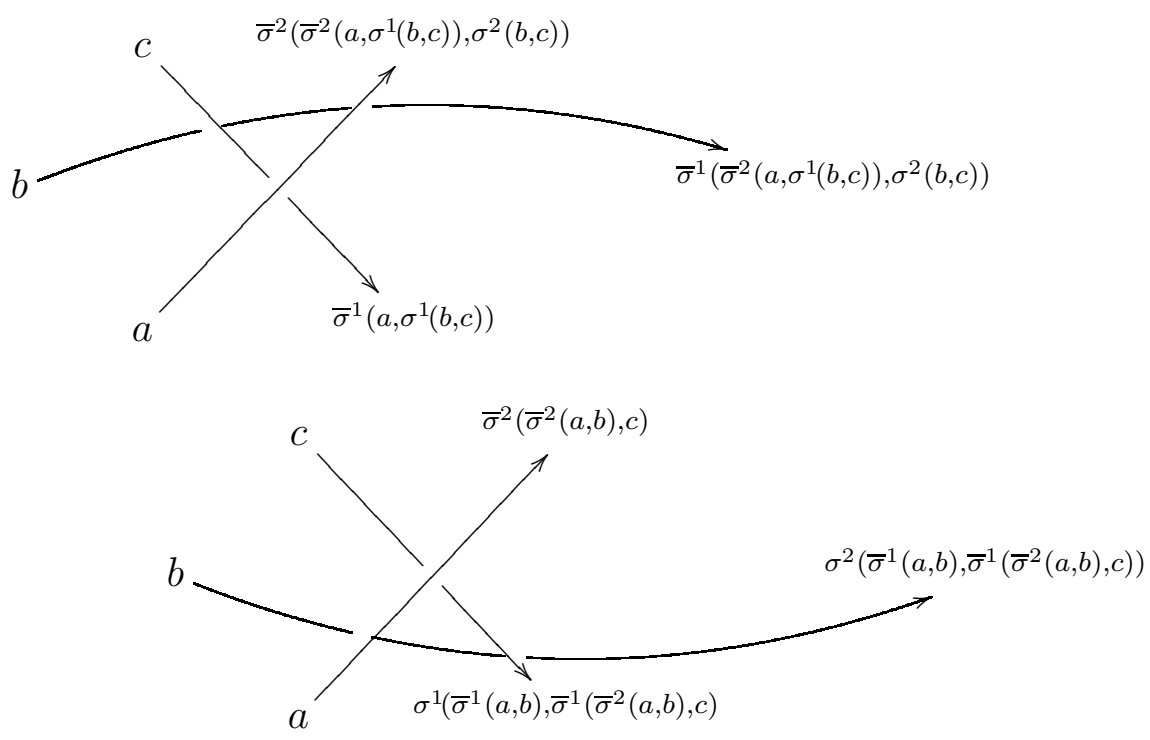

The product of weights for the horizontal line in the first diagram is

$$
I=f(b, c) f^{-1}\left(\bar{\sigma}\left(\bar{\sigma}^{2}\left(a, \sigma^{1}(b, c)\right), \sigma^{2}(b, c)\right)\right)
$$

and for the second diagram is

$$
I I=f^{-1}(\bar{\sigma}(a, b)) f\left(\bar{\sigma}^{1}(a, b), \bar{\sigma}^{1}\left(\bar{\sigma}^{2}(a, b), c\right)\right) .
$$

Using (11) in I:

$$
I=f(b, c) f\left(\left(\sigma^{2}\left(\bar{\sigma}^{1}(a, b), \bar{\sigma}^{1}\left(\bar{\sigma}^{2}(a, b), c\right)\right), \bar{\sigma}^{2}\left(\bar{\sigma}^{2}(a, b), c\right)\right)\right)
$$

Take te changes of variables $\left(x_{1}, d\right)=\bar{\sigma}(a, b)$ and $\left(x_{2}, x_{3}\right)=\bar{\sigma}(d, c)$. Then

$$
\begin{gathered}
I=f\left(\sigma^{2}\left(x_{1}, \sigma^{1}\left(x_{2}, x_{3}\right)\right), \sigma^{2}\left(x_{2}, x_{3}\right)\right) f^{-1}\left(\sigma^{2}\left(x_{1}, x_{2}\right), x_{3}\right) \\
I I=f^{-1}\left(x_{1}, \sigma^{1}\left(x_{2}, x_{3}\right)\right) f\left(x_{1}, x_{2}\right)
\end{gathered}
$$

We see that if $f$ is a non-commutative 2 cocycle then $I=I I$.

The weights that correspond to the other crossings are:

$$
I I I=f^{-1}\left(\bar{\sigma}\left(a, \sigma^{1}(b, c)\right)\right), I V=f^{-1}\left(\bar{\sigma}\left(\bar{\sigma}^{2}(a, b), c\right)\right),
$$

changing variables and composing (1) with $1 \times \delta$ :

$$
I I I=f^{-1}\left(\sigma^{1}\left(x_{1}, x_{2}\right), \sigma^{1}\left(\sigma^{2}\left(x_{1}, x_{2}\right), x_{3}\right)\right), I V=f^{-1}\left(x_{2}, x_{3}\right)
$$

$I I I=I V$ is verified as $f$ is a 2 -cocycle.

Case 4: We only exhibit the diagram corresponding to this case, the computations are similar to the previous case. 

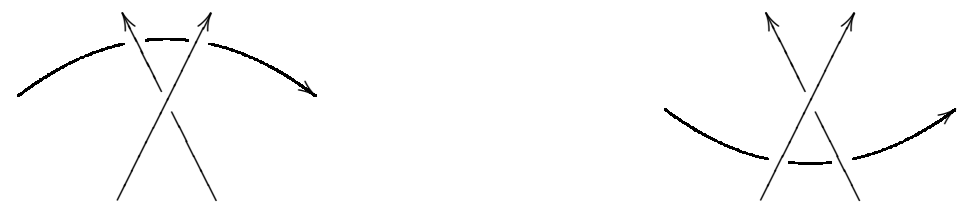

This shows, not only, that the product of the weights does not change under Reidemeister moves but the remaining weights stay the same.

For a group element $h \in H$, denote $[h]$ denote the conjugacy class to which $h$ belongs.

Definition 11. The set of conjugacy classes

$$
\left.\vec{\Psi}(L, f)=\vec{\Psi}_{(X, f)}(L)=\left\{\left[\Psi_{i}(L, \mathcal{C}, f)\right]\right\}\right\}_{\substack{1 \leq i \leq r \\ \mathcal{C} \in \operatorname{Col}_{X}(L)}}
$$

where $\Psi_{i}(L, \mathcal{C}, f)=\prod_{j=1}^{k(i)} B_{f}\left(\tau_{j}^{(i)}, \mathcal{C}\right)$ (the order in this product is following the orientation of the component) is called the conjugacy biquandle cocycle invariant of the link.

Theorem 12. The conjugacy biquandle cocycle invariant $\Psi$ is well defined.

Proof. The fact that $\Psi$ does not change under Reidemeister moves for fixed base points was proven earlier. A change of base points causes cyclic permutations of Boltzmann weights, and hence the invariant is defined up to conjugacy.

Proposition 13. If $f, g$ are two cohomologous non-commutative 2-cocyle functions then $\left[\Psi_{i}(L, \mathcal{C}, f)\right]=\left[\Psi_{i}(L, \mathcal{C}, g)\right]$

Proof. Let us suppose $f\left(x_{1}, x_{2}\right)=\gamma\left(x_{1}\right) g\left(x_{1}, x_{2}\right) \gamma^{-1}\left(\sigma^{2}\left(x_{1}, x_{2}\right)\right)$. There are four cases to analyse:

(1)

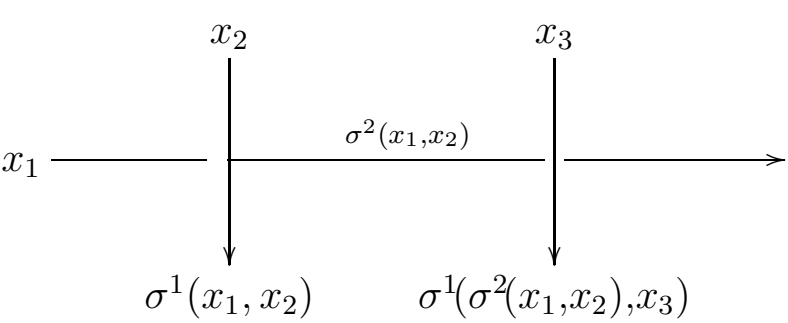

(3)

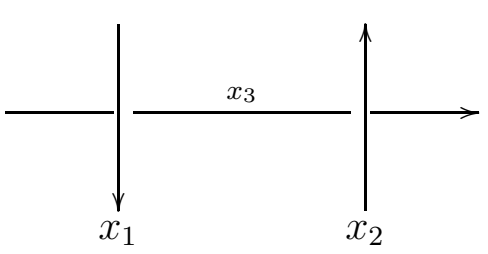

$(4)$
$(2)$
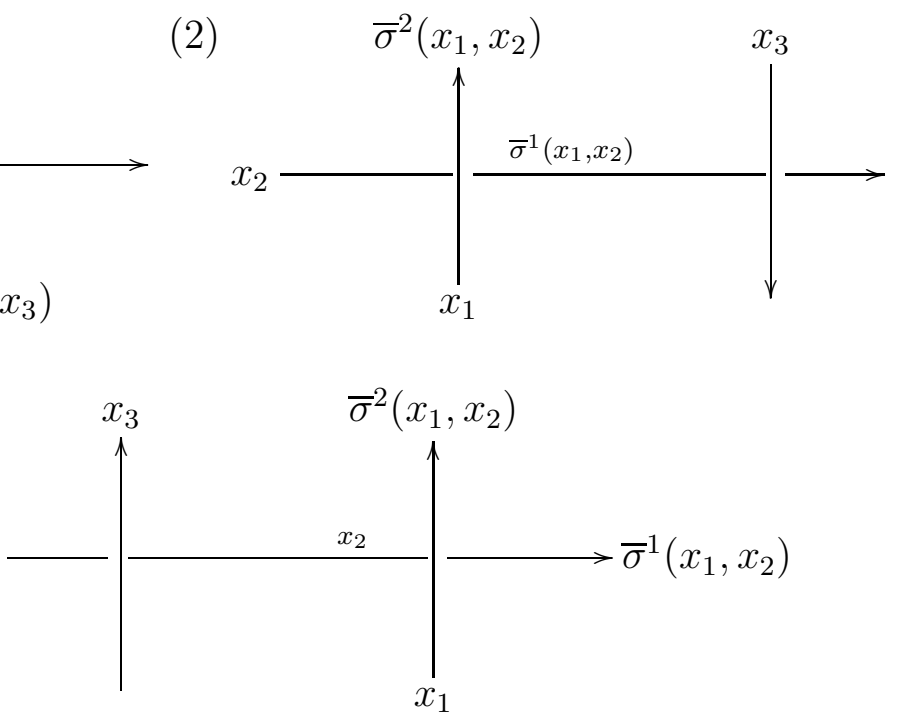

In case 1$)$, the product of weights for the horizontal line is: $f\left(x_{1}, x_{2}\right) f\left(\sigma^{2}\left(x_{1}, x_{2}\right), x_{3}\right)=$

$$
=\gamma\left(x_{1}\right) g\left(x_{1}, x_{2}\right) \gamma^{-1}\left(\sigma^{2}\left(x_{1}, x_{2}\right)\right) \gamma\left(\sigma^{2}\left(x_{1}, x_{2}\right) g\left(\sigma^{2}\left(x_{1}, x_{2}\right), x_{3}\right) \gamma^{-1}\left(\sigma^{2}\left(\sigma^{2}\left(x_{1}, x_{2}\right), x_{3}\right)\right)\right.
$$


In case 2$): f^{-1}\left(\bar{\sigma}\left(x_{1}, x_{2}\right)\right) f\left(\bar{\sigma}^{1}\left(x_{1}, x_{2}\right), x_{3}\right)=$ $\gamma\left(\sigma^{2}\left(\bar{\sigma}\left(x_{1}, x_{2}\right)\right)\right) g^{-1}\left(\bar{\sigma}\left(x_{1}, x_{2}\right)\right) \gamma^{-1}\left(\bar{\sigma}^{1}\left(x_{1}, x_{2}\right)\right) \gamma\left(\bar{\sigma}^{1}\left(x_{1}, x_{2}\right)\right) g\left(\bar{\sigma}^{1}\left(x_{1}, x_{2}\right), x_{3}\right) \gamma^{-1}\left(\sigma^{2}\left(\bar{\sigma}^{1}\left(x_{1}, x_{2}\right), x_{3}\right)\right)$

In case 3): $f\left(\bar{\sigma}\left(x_{1}, x_{3}\right)\right) f^{-1}\left(\bar{\sigma}\left(x_{2}, x_{3}\right)\right)=$

$$
\gamma\left(\bar{\sigma}^{1}\left(x_{1}, x_{2}\right)\right) g\left(\bar{\sigma}\left(x_{1}, x_{3}\right)\right) \gamma^{-1}\left(\sigma^{2}\left(\bar{\sigma}\left(x_{1}, x_{3}\right)\right)\right) \gamma\left(\sigma^{2}\left(\bar{\sigma}\left(x_{2}, x_{3}\right)\right)\right) g\left(\bar{\sigma}\left(x_{2}, x_{3}\right)\right) \gamma^{-1}\left(\bar{\sigma}\left(x_{2}, x_{3}\right)\right)
$$

And finally in case 4$): f^{-1}\left(x_{2}, x_{3}\right) f^{-1}\left(\bar{\sigma}\left(x_{1}, x_{2}\right)\right)=$

$$
\gamma\left(\sigma^{2}\left(x_{2}, x_{3}\right)\right) g^{-1}\left(x_{2}, x_{3}\right) \gamma^{-1}\left(x_{2}\right) \gamma\left(\sigma^{2}\left(\bar{\sigma}\left(x_{1}, x_{2}\right)\right)\right) g^{-1}\left(\bar{\sigma}\left(x_{1}, x_{2}\right)\right) \gamma^{-1}\left(\bar{\sigma}^{1}\left(x_{1}, x_{2}\right)\right)
$$

\section{Universal non commutative 2-cocycle}

Given a biquandle $(X, \sigma)$ and a group $H$, recall a non commutative 2-cocycle is a function $f: X \times X \rightarrow H$ satisfying

$$
f(x, y) f\left(\sigma^{2}(x, y), z\right)=f\left(x, \sigma^{1}(y, z)\right) f\left(\sigma^{2}\left(x, \sigma^{1}(y, z)\right), \sigma^{2}(y, z)\right)
$$

and

$$
f\left(\sigma^{1}(x, y), \sigma^{1}\left(\sigma^{2}(x, y), z\right)\right)=f(y, z)
$$

for any $x, y, z \in X$, and is called type $\mathrm{I}$ if in addition $f(x, s(x))=1$.

Definition 14. We define $U_{n c}=U_{n c}(X, \sigma)$, the Universal biquandle 2-cocycle group, as the group freely generated by symbols $(x, y) \in X \times X$ with relations

(Unc1) $(x, y)\left(\sigma^{2}(x, y), z\right)=\left(x, \sigma^{1}(y, z)\right)\left(\sigma^{2}\left(x, \sigma^{1}(y, z)\right), \sigma^{2}(y, z)\right)$

(Unc2) $\left(\sigma^{1}(x, y), \sigma^{1}\left(\sigma^{2}(x, y), z\right)\right)=(y, z)$

(Unc3) $(x, s(x))=1$

The following is immediate from the definitions:

Proposition 15. Let $X$ be a biquandle:

- Denote $[x, y]$ the class of $(x, y)$ in $U_{n c}$. The map

$$
\begin{aligned}
\pi: X \times X & \rightarrow U_{n c} \\
(x, y) & \mapsto[x, y]
\end{aligned}
$$

is a type I non commutative 2-cocycle.

- Let $H$ be a group and $f: X \times X \rightarrow H$ a type I non commutative 2-cocycle, then there exists a unique group homomorphism $\bar{f}: U_{n c} \rightarrow H$ such that $f=\bar{f} \pi$.

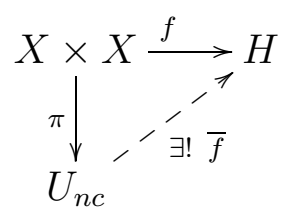


In particular, given $(X, \sigma)$, there exists non trivial 2-cocycles if and only if $U_{n c}$ is a nontrivial group.

Proposition 16. $U_{n c}$ is functorial. That is, if $\phi:(X, \sigma) \rightarrow(Y, \tau)$ is a morphism of set theoretical solutions of the YBeq, namely $\phi$ satisfy

$$
(\phi \times \phi) \sigma\left(x, x^{\prime}\right)=\tau\left(\phi x, \phi x^{\prime}\right)
$$

then, $\phi$ induces a (unique) group homomorphism $U_{n c}(X) \rightarrow U_{n c}(Y)$ satisfying

$$
\left[x, x^{\prime}\right] \mapsto\left[\phi x, \phi x^{\prime}\right]
$$

Proof. One need to prove that the assignment $\left(x, x^{\prime}\right) \mapsto\left(\phi x, \phi x^{\prime}\right)$ is compatible with the relations defining $U_{n c}(X)$ and $U_{n c}(Y)$ respectively, and that is clear since $(\phi \times \phi) \circ \sigma=$ $\tau \circ(\phi \times \phi)$.

Remark 17. In order to produce an invariant of a knot or link, given a solution $(X, \sigma)$, we need to produce a coloring of the knot/link by $X$, and then find a non commutative 2-cocycle, but since $U_{n c}$ is functorial, given $X$ we always have the universal 2-cocycle $X \times X \rightarrow U_{n c}(X)$, and hence, we only need to consider all different colorings.

Also, if $\phi: X \rightarrow X$ is a bijection commuting with $\sigma$, then, given a coloring and its invariant calculated with the universal cocycle, we may apply $\phi$ to each color and get another coloring, and this will produce the same invariant pushed by $\phi$ in $U_{n c}$.

Remark 18. Given a link $L$ of two strands colored using (both) colors $\{1,2\}$, the invariant obtained is $\Psi_{i}(L, \mathcal{C}, f)=(i, j)^{\ln (i, j)} i, j \in\{1,2\}$ and $i \neq j$, where $\ln (i, j)$ is the linking number between the two strands.

Proof. First notice that every component must be colored by a single color.

To every underarc of the component $i$ with itself will correspond a $(i, i)=1$ as weight. Then these crossings will not change the product. Then one can think that each component is unknoted with itself. It is well known that any two closed curves in space, if allowed to pass through themselves but not each other, can be moved a concatenation of the following standard positions:

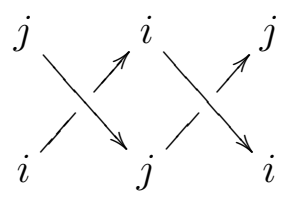

This diagram will contribute a factor $(i, j)^{\mathbf{1}}$ to $\Psi_{i}\left((j, i)\right.$ to $\left.\Psi_{j}\right)$ and if trying to calculate the linking number will add $\mathbf{1}$ for each pair of crossings. Analogously in the next diagram:

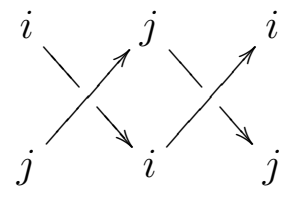

so, the invariant $\Psi_{i}$ will be $(i, j)^{\frac{a-b}{2}}=(i, j)^{\ln (i, j)}$ where $a, b$ are the total amount of positive and negative crossings

Example 19. The Whitehead link have linking number cero, the same happens taking the link consisting of two unknots. If you paint these links using $\operatorname{Wada}\left(\mathbb{Z}_{3}\right)$ (see example below), Whitehead has only 3 possibilities, while there are 9 ways to paint the pair of unknots. 


\subsection{Some examples of biquandles of small cardinality}

We first list some well-known general constructions generating biquandle solutions:

1. If $(X, \triangleleft)$ is a rack, one may consider two different solutions of the YBeq:

$$
\sigma(x, y)=(y, x \triangleleft y), \text { and } \bar{\sigma}(x, y):=\sigma^{-1}(x, y)=\left(y \triangleleft^{-1} x, x\right)
$$

these solutions are biquandles if and only if $(X, \triangleleft)$ is a quandle, namely $x \triangleleft x=x$ for all $x \in X$, in this case the function $s$ is the identity: $s(x)=x$.

When considering n.c. 2-cocycles, condition Unc2 is not preserved (in general) if one changes $\sigma$ with $\sigma^{-1}$, so it is relevant to see $\sigma$ and $\sigma^{-1}$ as different biquandles.

2. Let $\tau: X \times X \rightarrow X \times X$ denote the flip, namely $\tau(x, y)=(y, x)$. Let $\mu, \nu: X \rightarrow X$ be two bijections of $X$. then

$$
(\mu \times \nu) \tau(x, y)=(\mu(y), \nu(x))
$$

satisfies YBeq if and only if $\mu \nu=\nu \mu$, and this solution is a biquandle if and only if $\nu=\mu^{-1}$, in this case, the function $s: X \rightarrow X$ is equal to $\mu^{-1}$. In this way, the set of bijections of $X$ maps injectively into the set of biquandle structures on $X$, each conjugacy class of a given bijection maps into an isomorphism class of biquandle structures. Notice that every biquandle structure obtained in this way is involutive, namely $\sigma=\left(\mu \times \mu^{-1}\right) \circ \tau$ verifies $\sigma^{2}=\mathrm{Id}$.

3. Wada: if $G$ is a group, then the formula $\sigma(x, y)=\left(x y^{-1} x^{-1}, x y^{2}\right)$ is a biquandle, with $s(x)=x^{-1}$. As a particular case, if $G$ is abelian and with additive notation we have $\sigma(x, y)=(-y, x+2 y)$.

4. Alexander biquandle or Alexander switch:

Let $R$ be a ring, $s, t \in R$ two commuting units, and $M$ an $R$-module, then

$$
\sigma(x, y)=(s \cdot y, t \cdot x+(1-s t) \cdot y), \quad(x, y) \in M \times M
$$

is a biquandle, with function $s(x)=\left(s^{-1}\right) \cdot x$. In the particular case $s=-1, t=1$ one gets the abelian Wada's solution. If $s=1$ then one gets the solution induced by the Alexander rack.

These constructions give a lot of examples, but there are much more. If $|X|=2$, call $X=\{0,1\}$, one have the flip, satisfying $s(1)=1$ and $s(2)=2$, and this condition fully characterize this solution. If $s(0) \neq 0$ then $s(0)=1$ and necessarily $s(1)=0$; this forces $\sigma(0,0)=(1,1)$ and $\sigma(1,1)=(0,0)$. This is actually a biquandle coming from the bijection construction

$$
\sigma(x, y)=(y+1, x-1): x, y \in \mathbb{Z} / 2 \mathbb{Z}
$$

We will call this solution the antiflip.

If $|X|=3$, we call the elements $X=\{0,1,2\}$ and identify $X=\mathbb{Z} / 3 \mathbb{Z}$. The above constructions give the following list: 
1. There are three isomorphism classes of quandles of 3 elements:

(a) the trivial quandle $(x \triangleleft y=x$ for all $x, y)$, this gives the flip solution.

(b) $D_{3}: x \triangleleft y=2 y-x$, for $x, y \in \mathbb{Z} / 3 \mathbb{Z}$, which gives two solutions

$$
\sigma(x, y)=(y, x \triangleleft y)=(y, 2 y-x)
$$

and its inverse

$$
\bar{\sigma}(x, y)=\left(x \triangleleft^{-1} y, x\right)=(2 x-y, x)
$$

(c) another quandle which we call $Q_{3}$, with operation given by $-\triangleleft 0=(12)$ (the permutation $1 \leftrightarrow 2$ ), and $-\triangleleft 1=-\triangleleft 2=$ Id. The solution

$$
\sigma(x, y)=(y, x \triangleleft y)
$$

behaves like the flip for $x, y=1,2$, but

$$
\begin{aligned}
& \sigma(0,1)=(1,0), \sigma(1,0)=(0,2) \\
& \sigma(0,2)=(2,0), \sigma(2,0)=(0,1)
\end{aligned}
$$

One can check that this equalities can be achieved with the formula

$$
\sigma(x, y)=\left(y,-x-x y^{2}\right)=\left(y,-x\left(1+y^{2}\right)\right): x, y \in \mathbb{Z} / 3 \mathbb{Z}
$$

We also have the inverse solution.

2. If $X=\{0\} \coprod\{1,2\}$, with $\sigma(0, i)=(i, 0)$ and $\sigma(i, 0)=(0, i)$, then the flip on $\{1,2\}$ produces again the flip on three elements, but the other solution produce a new solution of the YBeq:

$$
\begin{gathered}
\sigma(1,2)=(1,2), \sigma(2,1)=(2,1) \\
\sigma(1,1)=(2,2), \sigma(2,2)=(1,1) \\
\sigma(0, i)=(i, 0), \sigma(i, 0)=(0, i)
\end{gathered}
$$

One may check that this equalities are given by $\sigma(x, y)=\left(y+x^{2} y, x+y^{2} x\right)$.

3. Wada's construction for $\mathbb{Z}_{3}$ gives the example $\sigma(x, y)=(-y, x-y)$ and its inverse: $\bar{\sigma}(x, y)=(y-x,-x)$.

4. Bijection biquandles:

(a) Using the bijection $\mu(x)=-x$ we have the solution $\sigma(x, y)=(-y,-x)$,

(b) if $\mu(x)=x+1$ then we have the solution $\sigma(x, y)=(y+1, x-1)$. One can check that all bijections $\neq$ Id are conjugated to one of these. 
For $M=R=\mathbb{Z}_{3}$, the units of $R$ are \pm 1 : the Alexander biquandle gives Wada's for $s=t=-1$, the Dihedral quandle solution for $s=1$ and $t=-1$, the flip for $s=t=1$, and the bijection solution $\sigma(x, y)=(-y,-x)$ when $s=t=-1$, so we have no new solution in this small cardinality considering the Alexander biquandle.

In this way, we obtain 10 solutions of the YBeq that are biquandles, three of them (flip, $Q_{3}$ and $D_{3}$ ) are quandle solutions. In A. Bartholomew and R. Fenn's classification list (see $[\mathrm{BF}]$ ) there are 7 biquandles that are not quandles, but we remark that, in Bartholomew and Fenn's list, if a solution $\sigma$ is listed, then $\bar{\sigma}$ is not listed, even thought $\sigma$ may not be isomorphic to $\bar{\sigma}$, as solution of the Yang-Baxter equation. For instance, for every quandle $(X, \triangleleft)$, the solution with "name" $X$ is $\sigma(x, y)=(y, x \triangleleft y)$ but the inverse solution $\sigma(x, y)=\left(x \triangleleft^{-1} y, x\right)$ do not appear in the list. We use the notation $B Q_{i}^{3}$, $i=1, \ldots, 10$ for the biquandles solutions of Bartholomew and Fenn, and we denote $B Q_{i}^{3 *}$ the inverse solution with respect to $B Q_{i}^{3}$.

\subsection{Computations of $U_{n c}$}

We begin with an explicit computation of the group $U_{n c}$ for a particular example; Wada: $\sigma(x, y)=(-y, x-y)$, which is also BiAlexander with $M=\mathbb{Z} / 3 \mathbb{Z}, s=-1, t=1$.

The fixed points are $(x, s(x))=(x,-x)$, that is $(0,0),(1,2),(2,1)$, so $(0,0)=(1,2)=$ $(2,1)=1$. Conditions (Unc1-2) are:

$$
\begin{gathered}
(x, y)(x-y, z)=(x,-z)(x+z, y-z) \\
(-y,-z)=(y, z)
\end{gathered}
$$

From the second equality we get generators $a=(0,1)=(0,-1), b=(1,0)=(-1,0)$ and $c=(1,1)=(-1,-1)$.

From the first cocycle equation

$$
(x, y)(x-y, z)=(x,-z)(x+z, y-z)
$$

if $y=s x$ (using $(x, s x)=1$ and also using Unc2) we get a trivial equality. If $y=s z$ we also get trivial equality, so in principle we have $3^{3}-9-9+3=12$ equations. If we write them all in terms of $a, b$ and $c$ we get

$$
\begin{gathered}
1=1, a=a, a b=a, a c=a c, b^{2}=b^{2}, b c=c, \\
1=1, c=c, b=b, 1=1, c a=c a, c a=b
\end{gathered}
$$

of course we can see trivial equations, if we exclude them we get

$$
a b=a, b c=c, c a=b
$$

from the first (and also the second) equation we can see that te generator $b$ is trivial. If we write again all the equations with the replacement $b=1$ we get

$$
c a=1
$$

We conclude $U_{n c}=\operatorname{Free}(a, c) /(a c=1) \cong \operatorname{Free}(a)$, but also we have described a procedure that can be implemented in a computer program: 
1. Add to the set $X \times X$ a new element "1" and begin to define an equivalence relation $(x, s(x)) \sim 1$.

2. from the second condition, add $(y, z) \sim\left(\sigma^{1}(x, y), \sigma^{1}\left(\sigma^{2}(x, y), z\right)\right)$ to the equivalence relation.

More precisely, given a list of subsets of $(X \times X) \coprod\{1\}$ whose union is $(X \times X) \coprod\{1\}$ (if this is not the case we add the sets $\{(x, y)\}$ to the list) one can easily give an algorithm producing the partition of $(X \times X) \cup\{1\}$ corresponding to the equivalence relation generated by the list of subsets: for each pair of subsets of the list, with nontrivial intersection, we replace these two subsets by their union, run over all different pairs, and iterate until saturate. We call classes this list of subsets.

3. From the data classes, choose representatives (if the list of subsets is ordered and their members are ordered, just pick the first member for each element of the list). Write down all cocycle equations, in terms of these representatives.

4. Eliminate the trivial equations, and

- for any cocycle equation where 1 appears, in case one found $a .1$, replace it by 1.a, so we do not count twice the same equation.

- For any cocycle equation of the form $a c=b c$ or $c a=c b$, add $a \sim b$ and recalculate the equivalence relation that it generates.

With the new data classes go to step 3, and iterate the process until it stabilizes.

The set in classes containing 1 is called $S$, this is a list of trivial elements in $U_{n c}$. A set of representatives of the others element of classes give a set of generators of $U_{n c}$. The remaining nontrivial 2-cocycle equations, written in terms of these representatives, give a set of relations. This algorithm produce a relatively small set of generators, and all the relations between them. For instance, in the example above will produce $U_{n c}=$ Free $(a, b) /(a b=1)$. We have implemented this algorithm in G.A.P, and it produces the following:

For the dihedral quandle $D_{3}$ :

Set of generators: $\left\{f_{1}=[1,2], f_{2}=[1,3], f_{3}=[2,1], f_{4}=[2,3], f_{5}=[3,1], f_{6}=[3,2]\right\}$. Trivial elements $S: 1=[1,1]=[2,2]=[3,3]$. Relations:

$$
f_{1} f_{5}=f_{2}, f_{2} f_{3}=f_{1}, f_{3} f_{6}=f_{4}, f_{4} f_{1}=f_{3}, f_{5} f_{4}=f_{6}, f_{6} f_{2}=f_{5}
$$

For Wada, the set of generators is $\left\{f_{1}=[1,2]=[1,3], f_{2}=[2,2]=[3,3]\right\}$. Trivial elements: $1=[1,1]=[2,1]=[2,3]=[3,1]=[3,2]$. Relations:

$$
f_{2} f_{1}=1
$$

An important remark on notation: G.A.P. always gives a numbering of the elements of its objects (and in particular, one cal always order them), for instance, $\mathbb{Z} / 3 \mathbb{Z}=\{\overline{0}, \overline{1}, \overline{2}\}$ has three elements, that G.A.P. number as $[1,2,3]$, where 1 is the first element, 2 is the second and so on; in order to identify the element one has to see the label, and 
in this case 1 corresponds to $\overline{0}, 2$ corresponds to $\overline{1}$ and 3 to $\overline{2}=\overline{-}$, so the equation $1=[1,1]=[2,1]=[2,3]=[3,1]=[3,2]$ means

$$
1=(\overline{0}, \overline{0})=(\overline{1}, \overline{0})=(\overline{1}, \overline{2})=(\overline{2}, \overline{0})=(\overline{2}, \overline{1})
$$

The new element "1" that we add to $X \times X$ is called [ ], so for example the equality $1=[1,1]=[2,1]=[2,3]=[3,1]=[3,2]$ comes from the fact that the list (of lists) classes contains the element $[[],[1,1],[2,1],[2,3],[3,1],[3,2]]$.

In the following we use the notation as in G.A.P.

For the inverse solution to Wada's: generators $\left\{f_{1}=[1,2]=[2,1]=[3,3], f_{2}=\right.$ $[1,3]=[2,2]=[3,1]\}$, trivial elements: $1=[1,1]=[2,3]=[3,2]$, relations:

$$
f_{2} f_{2}=f_{1}, f_{1} f_{1}=f_{2}
$$

For the flip on 2 elements $\{1,2\}$ : generators $\left\{f_{1}=[1,2], f_{2}=[2,1],\right\}$, trivial elements: $1=[1,1]=[2,2]$, and no equations at all.

For the flip in 3 elements $\{1,2,3\}$ : generators: $\left\{f_{1}=[1,2], f_{2}=[1,3], f_{3}=[2,1], f_{4}=\right.$ $\left.[2,3], f_{5}=[3,1], f_{6}=[3,2]\right\}$, trivial elements: $1=[1,1]=[2,2]=[3,3]$, relations: $f_{2} f_{1}=f_{1} f_{2}, f_{4} f_{3}=f_{3} f_{4}, f_{6} f_{5}=f_{5} f_{6}$.

Taking the list of biquandles of cardinality 3 from Bartholomew and Fenn's list, adding the inverse solutions (when they are not isomorphic), we obtain the table below.

We remark that the procedure gives not only the number of generators, but the full equivalence class, we omit the full data in the table just for space considerations. We also add to the table the order of $\sigma$, and the number of fixed points on the diagonal

\begin{tabular}{|c|c|c|c|c|c|}
\hline name & $\sigma$ & $\begin{array}{l}\text { generators } \\
\text { of } U_{n c}\end{array}$ & equations & $\begin{array}{l}\text { order } \\
\text { of } \sigma\end{array}$ & $\# \Delta^{\sigma}$ \\
\hline flip & $B Q_{1}^{3}$ & 6 & $\begin{array}{c}f_{2} f_{1}=f_{1} f_{2}, f_{4} f_{3}=f_{3} f_{4}, \\
f_{6} f_{5}=f_{5} f_{6},\end{array}$ & 2 & 3 \\
\hline \multirow[t]{3}{*}{$a-f l i p \cup\{1\}$} & $B Q_{2}^{3}$ & 3 & $f_{3} f_{2}=f_{2} f_{3}$ & 2 & 1 \\
\hline & $B Q_{3}^{3}$ & 3 & - & 4 & 1 \\
\hline & $B Q_{3}^{3 *}$ & 3 & - & 4 & 1 \\
\hline Wada $\left(\mathbb{Z}_{3}\right)$ & $B Q_{4}^{3}$ & 2 & $f_{2} f_{1}=1$ & 3 & 1 \\
\hline \multirow[t]{2}{*}{ inv. $\operatorname{Wada}\left(\mathbb{Z}_{3}\right)$} & $B Q_{4}^{3 *}$ & 2 & $f_{1} f_{1}=f_{2}, f_{2} f_{2}=f_{1}$ & 3 & 1 \\
\hline & $B Q_{5}^{3}$ & 3 & $f_{2} f_{1}=f_{1} f_{2}$ & 2 & 3 \\
\hline$Q_{3}$ & $B Q_{6}^{3}$ & 3 & - & 4 & 3 \\
\hline inverse $Q_{3}$ & $B Q_{6}^{3 *}$ & 3 & - & 4 & 3 \\
\hline$(x, y) \mapsto(-y,-x)$ & $B Q_{7}^{3}$ & 3 & $f_{3} f_{2}=f_{2} f_{3}$ & 2 & 1 \\
\hline$D_{3}$ & $B Q_{8}^{3}$ & 6 & $\begin{array}{l}f_{1} f_{5}=f_{2}, f_{2} f_{3}=f_{1}, f_{3} f_{6}=f_{4}, \\
f_{4} f_{1}=f_{3}, f_{5} f_{4}=f_{6}, f_{6} f_{2}=f_{5},\end{array}$ & $\begin{array}{l}3 \\
3\end{array}$ & 3 \\
\hline \multirow[t]{3}{*}{ inverse $D_{3}$} & $B Q_{8}^{3 *}$ & 0 &,- & 3 & 3 \\
\hline & $B Q_{9}^{3}$ & 2 & $f_{2} f_{2}=f_{1}, f_{1} f_{1}=f_{2}$, & 3 & 0 \\
\hline & $B Q_{9}^{3 *}$ & 0 & - & 3 & 0 \\
\hline involutive $\left(\mathbb{Z}_{3}\right)$ & $B Q_{10}^{3}$ & 2 & $f_{1} f_{2}=f_{2} f_{1}$ & 2 & 0 \\
\hline
\end{tabular}
$\Delta:=\{(x, x): x \in X\}$, for instance, $\Delta^{\sigma}=\Delta$ if $X$ is a quandle.

We remark that for some cases (i.e. $B Q_{4,8,9}$ ) the invariant $U_{n c}$ distinguish between $\sigma$ and $\bar{\sigma}$. For $B Q_{3}^{3}$, the generators are the same in the strong sense that the equivalent 
classes of generators (as equivalent classes in $X \times X$ ) are the same, the relations are also the same (no relation at all), so they will give the same knot/link invariants, even though $\sigma$ and $\bar{\sigma}$ are non isomorphic biquandle solutions.

For most of the cases there is no much more to say in order to describe $U_{n c}$ as a group, for instance $U_{n c}($ flip $)=U_{n c}\left(B Q_{1}^{3}\right)=\mathbb{Z}^{2} * \mathbb{Z}^{2} * \mathbb{Z}^{2}$ : the free product of three copies of $\mathbb{Z}^{2}$, $U_{n c}\left(B Q_{3}^{3}\right) \cong U_{n c}\left(B Q_{6}^{3}\right) \cong F_{3}$ : the free group on 3 generators, $U_{n c}\left(B Q_{2}^{3}\right) \cong U_{n c}\left(B Q_{5}^{3}\right) \cong$ $U_{n c}\left(B Q_{7}^{3}\right) \cong \operatorname{Free}(a, b, c) /(b c=c b), U_{n c}\left(B Q_{1}^{3} 0\right) \cong \mathbb{Z}^{2}$. . On the other hand, there are some simplifications for the remaining cases:

$$
\begin{gathered}
U_{n c}\left(B Q_{4}^{3}\right) \cong \operatorname{Free}(a, b) /(a b=1)=\operatorname{Free}(a) \cong \mathbb{Z} \\
U_{n c}\left(B Q_{9}^{3}\right) \cong B Q_{4}^{3 *} \cong \operatorname{Free}(a, b) /\left(a^{2}=b, b^{2}=a\right) \cong \operatorname{Free}(a) /\left(a^{3}=1\right) \\
U_{n c}\left(D_{3}\right) U_{n c}\left(B Q_{8}^{3}\right)=F_{6} /\left(f_{1} f_{5}=f_{2}, f_{2} f_{3}=f_{1}, f_{3} f_{6}=f_{4}, f_{4} f_{1}=f_{3}, f_{5} f_{4}=f_{6}, f_{6} f_{2}=f_{5}\right)
\end{gathered}
$$

Call $a:=f_{1}, b:=f_{5}$ and $c:=f_{4}$, we have

$$
a b=f_{2}, f_{2} f_{3}=a, f_{3} f_{6}=c, c a=f_{3}, b c=f_{6}, f_{6} f_{2}=b
$$

in particular, we can solve $f_{2}, f_{3}$ and $f_{6}$ in terms of $a, b, c$, so $U_{n c}$ is generated by $a, b, c$. In order to know the relations, we replace $f_{2}=a b, f_{3}=c a$ and $f_{6}=b c$ in the above equations and get

$$
a b c a=a, c a b c=c, b c a b=b
$$

or equivalently

$$
a b c=1, c a b=1, b c a=1
$$

whose solution is $c=(a b)^{-1}$. We conclude $U_{n c}\left(D_{3}\right)=\operatorname{Free}(a, b)$.

The computer program gives the set of generators and relations in a reasonable human time for biquandles of cardinality 12 or less. As a matter of numerical experiment, the groups associated to the inverse solution of biAlexander solution on $\mathbb{Z}_{m}$, for $s=-1$, and $t=1$, are cyclic of order $m$ (in a non trivial way) if $m=3,5,7,11,13$ (and much more complicated groups for $m=4,6,8,9,10,12)$. We don't know if this is a general fact for all primes $p$. There are nevertheless some general results that can be prove without computer:

\section{Inverse quandle solutions}

If $(X, \triangleleft)$ is a quandle then $\sigma(x, y)=(y, x \triangleleft y)$ is a biquandle, and condition Unc2 is trivial. But if we consider the inverse solution: $\bar{\sigma}(x, y)=\left(y \triangleleft^{-1} x, x\right)$ then condition (Unc2) is not trivial, we have the relations

$$
\begin{gathered}
(x, y)(x, z)=\left(x, z \triangleleft^{-1} y\right)(x, y) \\
\left(y \triangleleft^{-1} x, z \triangleleft^{-1} x\right)=(y, z) \\
(x, x)=1
\end{gathered}
$$

We see that, in presence of the second identity, the first one can be modified into

$$
(x, y)(x, z)=(x \triangleleft y, z)(x, y) \quad(\mathrm{Unc} \overline{\mathrm{Q}})
$$


or also $(x \triangleleft z, y \triangleleft z)(x, z)=(x \triangleleft y, z)(x, y)$. Notice that (Unc $\bar{Q})$, with $x=z$, says

$$
(x, y)(x, x)=(x \triangleleft y, x)(x, y) \Rightarrow(x, y)=(x \triangleleft y, x)(x, y) \Rightarrow 1=(x \triangleleft y, x)
$$

This equation, for $x=y$, gives $1=(x \triangleleft x, x)=(x, x)$. That is, $1=(x \triangleleft y, x)$ implies the type I condition. So, we may list a set of relations for $U_{n c}(\bar{\sigma})$ in the following way

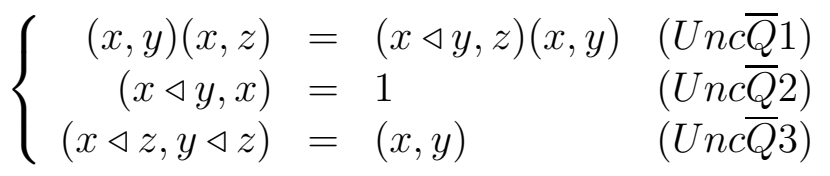

Corollary 20. Let $Q$ be a quandle and consider the biquandle solution $\bar{\sigma}(a, b)=\left(b \triangleleft^{-1}\right.$ $a, a)$. If $Q$ is such that for every $z \in Q$ there exists $y$ with $z=x \triangleleft y$, then $U_{n c}(\bar{\sigma})=1$.

Proof. Given $(z, x)$, let $y$ be such that $z=x \triangleleft y$, then $(z, x)=(x \triangleleft y, x)=1$.

Example 21. If $(X, \triangleleft)=D_{n}=(\mathbb{Z} / n \mathbb{Z}, x \triangleleft y=2 y-x)$ with $n$ is odd then $U_{n c}(\bar{\sigma})=1$.

\section{The reduced $U_{n c}$}

We recall that if $f: X \times X \rightarrow G$ is a (type I) cocycle and $\gamma: X \rightarrow G$ is a function satisfying $\gamma(x)=\gamma(s x)$, then $f_{\gamma}(x, y):=\gamma(x) f(x, y) \gamma\left(\sigma^{2}(x, y)\right)^{-1}$ is also a (type I) 2cocycle, and the knot/link invariant produce by $f$ is the same as the one produced by $f$. In particular, one can consider the universal 2-cocycle $\pi: X \times X \rightarrow U_{n c}$ and try to see if there is a cohomologous one, simpler that $\pi$. This procedure leads to a construction that we call reduced universal group:

Definition 22. Let $\gamma: X \rightarrow U_{n c}$ be a (set theoretical) map such that $\gamma(x)=\gamma(s(x))$ and $\pi_{\gamma}: X \times X \rightarrow U_{n c}$ given by

$$
\pi_{\gamma}(x, y)=\gamma(x)(x, y) \gamma\left(\sigma^{2}(x, y)\right)^{-1}
$$

Define $S=\left\{(x, y) \in X \times X: \pi_{\gamma}(x, y)=1 \in U_{n c}\right\} \subseteq X \times X$ and consider the group $U_{n c}^{\gamma}$ defined by

$$
U_{n c}^{\gamma}:=U_{n c} /<\pi(x, y) /(x, y) \in S>
$$

Denote $\overline{[x, y]} \in U_{n c}^{\gamma}$ the class of $(x, y)$ and $p: X \times X \rightarrow U_{n c}^{\gamma}$ the map $p(x, y)=\overline{[x, y]}$.

Theorem 23. With notations as in the above definition, The map $p: X \times X \rightarrow U_{n c}^{\gamma}$ has the following universal property:

- $p$ is a 2-cocycle.

- for any group $G$ and 2-cocycle $f: X \times X \rightarrow G$, there exists a cohomologous map $f^{\Gamma}$ and a group homomorphism $f^{\Gamma}: U_{n c}^{\gamma} \rightarrow G$ such that $f^{\Gamma}$ factorizes through $p$, that is $f^{\Gamma}=f^{\Gamma} p$. 
Proof. The fact that $p$ is a 2-cocycle is immediate. By (15) we obtain the existence of the unique group morphism $\bar{f}$ such that

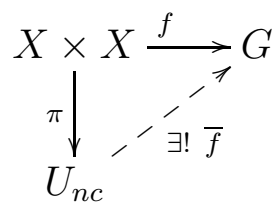

commutes. Define $f^{\Gamma}:=\bar{f} \circ \pi_{\gamma}$; in diagram: $X \times \underset{\pi_{\gamma}}{\longrightarrow} U_{n c}$

We have

$$
f^{\Gamma}(x, y)=\bar{f} \circ \pi_{\gamma}(x, y)=\bar{f} \circ \gamma(x) \bar{f} \circ \pi(x, y)\left(\bar{f} \circ \gamma\left(\sigma^{2}(x, y)\right)\right)^{-1}
$$

so $f^{\Gamma}$ and $\bar{f} \circ \pi=f$ are cohomologous. Using again the universal property of $U_{n c}, f^{\Gamma}$ factorizes through $U_{n c}$, hence there exists a group homomorphism $\overline{f^{\Gamma}}: U_{n c} \rightarrow G$ such that $f^{\Gamma}=\overline{f^{\Gamma}} \circ \pi$.

On the other hand, since $\pi_{\gamma}(S)=1$ we have $f^{\Gamma}(S)=\bar{f}\left(\pi_{\gamma}(S)\right)=\bar{f}(1)=1$, but also $f^{\Gamma}(S)=\overline{f^{\Gamma}}(\pi(S))$, so the group homomorphism $\overline{f^{\Gamma}}: U_{n c} \rightarrow G$ induces a map $f^{\gamma} U_{n c}^{\gamma}=$ $U_{n c} / \pi(S) \rightarrow G$ such that, if $p^{\prime}: U_{n c} \rightarrow U_{n c} / \pi(S)$ is the canonical group projection to the quotient $\left(p=p^{\prime} \circ \pi\right)$, then $\overline{f^{\Gamma}}=f^{\gamma} \circ p^{\prime}$. In diagram:

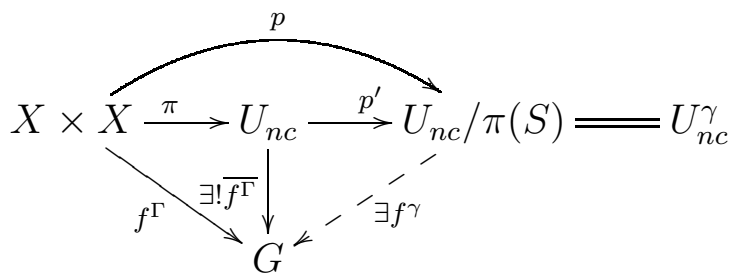

Clearly $f^{\Gamma}=f^{\gamma} \circ p$.

For a given $\gamma$, the associated $U_{n c}^{\gamma}$ is called the reduced universal group.

Corollary 24. Given a biquandle $X$, if there exists $\gamma: X \rightarrow U_{n c}$ such that $U_{n c}^{\gamma}=1$ then every 2-cocycle in $X$ is trivial.

A general example of the above situation is given by some Alexander biquandles.

\subsection{The Alexander biquandle}

Let $A=\mathbb{Z}\left[s, t, s^{-1}, t^{-1}\right], X$ an $A$-module and $\sigma: X \times X \rightarrow X \times X$ given by the matrix

$$
\left(\begin{array}{cc}
0 & t \\
s & (1-s t)
\end{array}\right)
$$

equivalently $\sigma(x, y)=(s y, t x+(1-s t) y)$. The condition of being a fixed point is $x=s y$ :

$$
\sigma(s y, y)=((s y), t(s y)+(1-s t) y)=(s y, y)
$$


Cocycle conditions are

$$
\left\{\begin{aligned}
(x, y)(t x+(1-s t) y, z) & =(x, s z)(t x+(1-s t) s z, t y+(1-s t) z) \\
(s y, s z) & =(y, z) \\
(s y, y) & =1
\end{aligned}\right.
$$

Following M. Graña, we can adapt to the biquandle situation the proof for the quandle case (see Lemma 6.1 of [G]). Consider $\gamma: X \rightarrow U_{n c}$ given by $\gamma(x)=(0, c x)$, where $c=(1+s t)^{-1}$. Notice that $c$ is an endomorphism commuting with $s$, and $(s y, s z)=$ $(y, z) \in U_{n c}$, so

$$
\gamma(x)=(0, c x)=(s 0, s c x)=(0, c s x)=\gamma(s x)
$$

hence, we can use $\gamma$ in order to get another 2-cocycle, cohomologous to $\pi$. Recall

$$
\pi_{\gamma}(x, y):=\gamma(x)(x, y) \gamma\left(\sigma^{2}(x, y)\right)^{-1}
$$

where $\sigma(x, y)=(s y, t x+(1-s t) y)$, so

$$
\pi_{\gamma}(x, y)=(0, c x)(x, y)(0, c(t x+(1-s t) y))^{-1}
$$

in particular

$$
\pi_{\gamma}(0, y)=(0,0)(0, y)(0, c(1-s t) y)^{-1}=(0, y)(0, y)^{-1}=1
$$

so, the class of $(0, y)$ is trivial in $U_{n c}^{\gamma}$.

Lemma 25. Let $X$ be an Alexander birack such that $(1-s t)$ is invertible in $\operatorname{End}(X)$. If we define $\gamma$ as above, then, the following identities hold in $U_{n c}^{\gamma}$ :

1. $(x, 0)=1$ for all $x$, and

2. $(a, b)=(a, b+a)$ for all $a, b \in X$.

Proof. 1. From the cocycle condition,

$$
(x, y)(t x+(1-s t) y, z)=(x, s z)(t x+(1-s t) s z, t y+(1-s t) z)
$$

taking $x=0=z$ we get

$$
(0, y)((1-s t) y, 0)=(0,0)(0, t y)
$$

but we know that $(0, *)=1$ in $U_{n c}^{\gamma}$, so $((1-s t) y, 0)=1$, and because $(1-s t)$ is a unity we conclude $(x, 0)=1$ for all $x$.

2. Using the cocycle condition

$$
(x, y)(t x+(1-s t) y, z)=(x, s z)(t x+(1-s t) s z, t y+(1-s t) z)
$$

and clear $z$ from $t x+(1-s t) s z=0$, that is, set $z=\frac{-t}{(1-s t) s} x$, then

$$
(x, y)\left(t x+(1-s t) y, \frac{-t}{(1-s t) s} x\right)=\left(x, s \frac{-t}{(1-s t) s} x\right)(0, t y+(1-s t) z)
$$


or

$$
(x, y)\left(t x+(1-s t) y, \frac{-t}{(1-s t) s} x\right)=\left(x, \frac{-t}{(1-s t)} x\right)
$$

clearing $y=\frac{-t}{1-s t} x$, get

or

$$
\left(x, \frac{-t}{1-s t} x\right)(0, z)=(x, s z)\left(t x+(1-s t) s z, t \frac{-t}{1-s t} x+(1-s t) z\right)
$$

$$
\left(x, \frac{-t}{1-s t} x\right)=(x, s z)\left(t x+(1-s t) s z, \frac{-t^{2}}{1-s t} x+(1-s t) z\right)
$$

in particular, using RHS of $(*)=\operatorname{LHS}$ of $(\dagger)$ with $y=s z$ we get

$$
(x, s z)\left(t x+(1-s t) s z, \frac{-t}{(1-s t) s} x\right)=(x, s z)\left(t x+(1-s t) s z, \frac{-t^{2}}{1-s t} x+(1-s t) z\right)
$$

$\mathrm{SO}$

$$
\left(t x+(1-s t) s z, \frac{-t}{(1-s t) s} x\right)=\left(t x+(1-s t) s z, \frac{-t^{2}}{1-s t} x+(1-s t) z\right)
$$

Now we simply change variables. Call $a=t x+(1-s t) s z$, then $(1-s t) z=a-\frac{t}{s} x$, replacing

Or

$$
\left(a, \frac{-t}{(1-s t) s} x\right)=\left(a, \frac{-t^{2}}{1-s t} x+a-\frac{t}{s} x\right)
$$

$$
\left(a, \frac{-t}{(1-s t) s} x\right)=\left(a, \frac{-t}{(1-s t) s} x+a\right)
$$

Call $b=\frac{-t}{(1-s t) s} x$ (notice that $(x, y) \mapsto(a, b)$ is bijective) and get $(a, b)=(a, b+a)$.

Inductively $(a, b)=(a, b+n a) \forall n \in \mathbb{N}$; if $a$ generates $X$ additively then

$$
(a, b)=(a, 0)=1 \forall b \in X
$$

Corollary 26. If $p$ is an odd prime, $X=\mathbb{F}_{p}$, and $s^{-1} \neq t \in \mathbb{F}_{p} \backslash\{0\}$, then every cocycle in the Alexander's birack in $X$ is cohomologous to the trivial one. In other words, the reduced Universal group $U_{n c}^{\gamma}$ is trivial. In particular every 2-cocycle in $D_{3}$ is trivial.

Remark 27. This generalizes the result of Graña in [G] where he proves the quandle case, that is, the case $s=1$.

A biquandle example that is not a quandle is the following:

Corollary 28. Let $X=\mathbb{Z}_{3}$, then Wada's biquandle agree with biAlexander biquandle $\left(s=-1=t, 1-s t=-1 \in \mathbb{Z}_{3}\right)$, so $U_{n c}^{\gamma}=1$ and every non commutative 2-cocycle is trivial. In particular, for any coloring with this biquandle, the corresponding element in $U_{n c}$ is trivial.

Remark 29. For the inverse solution $\bar{\sigma}$ of of Wada's biquandle (with $G=\mathbb{Z}_{3}$ ), the group $U_{n c}(\bar{\sigma})=\left\langle a: a^{3}=1\right\rangle$ is not trivial. One may wander if there is a function $\gamma$ such that $U_{n c}^{\gamma}(\bar{\sigma})$ is the trivial group, but this is not the case. We will see examples where the invariant obtained using Wada's inverse solution is not trivial, actually, it distinguishes the trefoil from its mirror image.

Remark 30. In the previous corollary, the hypothesis $|X|$ being prime was essential, the smallest case where it fails is $X=\mathbb{Z}_{4}$, as an example of computation we calculate the reduced universal group for $X=\mathbb{Z}_{4}$ and for $Z_{8}$ with $s=-1$ and $t=1$. 


\subsection{Reduced $U_{n c}$ in computer}

It is clear that the procedure that computes $U_{n c}$ in the computer can be trivially adapted for the reduced version, just adding as input a given set $S_{0} \subset X \times X$, and begin with $S=S_{0} \cup\{(x, s x): x \in S\}$, instead of simply $S=\{(x, s x): x \in S\}$. The procedure will actually compute a list of generators and relations of the quotient group $U_{n c} /\left(S_{0}\right)$, so it could be also used to produce other quotients, not only $U_{n c}^{\gamma}$. The advantage of $U_{n c}^{\gamma}$ is that it gives the same knot/link invariant as $U_{n c}$, so in order to find suitable $S_{0}$ 's one can do the following:

- In order to produce functions $\gamma: X \rightarrow U_{n c}$ with $\gamma(x)=\gamma(s x)$, consider the equivalence relation on $X$ induced by $s$, that is the equivalence relation generated by $x \sim s(x)$. Denote $\bar{x}$ the class of $x$ modulo $s$.

- for all pairs $(x, y) \in X \times X$, consider the coboundary relation

$$
f_{\gamma}(x, y)=\gamma(x) f(x, y) \gamma\left(\sigma^{2}(x, y)\right)^{-1}
$$

if $\bar{x}=\overline{\sigma^{2}(x, y)}$ then $f(x, y)$ is conjugated to $f_{\gamma}(x, y)$, so $f(x, y)=1 \Longleftrightarrow f_{\gamma}(x, y)=$ 1 , so it is clear that $(x, y)$ can not be included in $S$ because of $\gamma$.

- if $\bar{x} \neq \overline{\sigma^{2}(x, y)}$ then we can choose $\gamma: X \rightarrow U_{n c}$ such that $\gamma(z)=\gamma(s z)$ for all $z$ and $\gamma(x) f(x, y)=\gamma\left(\sigma^{2}(x, y)\right)$.

By the above considerations, it is useful to list all tuples

$$
\left(\bar{x},(x, y), \overline{\sigma^{2}(x, y)}\right)
$$

with $\bar{x} \neq \overline{\sigma^{2}(x, y)}$. For any of these elements, add the pair $\left(\bar{x}, \overline{\sigma^{2}(x, y)}\right)$ to a set $\mathrm{f}$ "used" elements, so we continue with the others with $\left(\bar{x},(x, y), \overline{\sigma^{2}(x, y)}\right)$ with $\bar{x} \neq \overline{\sigma^{2}(x, y)}$ but $\left(\bar{x}, \overline{\sigma^{2}(x, y)}\right)$ not "used". This procedure is easily implemented in G.A.P. For example, for the Dihedral quandle gives

$$
[0,[0,1], 2],[0,[0,2], 1],[1,[1,0], 2]]
$$

so we can choose $\gamma(0)=1, \gamma(2)=[0,1], \gamma(1)=[0,2]$ and hence define $S_{0}:=\{[0,1],[0,2]\}$. With this entry, the procedure computing $U_{n c}^{\gamma}$ gives $S=X \times X$, that is $U_{n c}^{\gamma}\left(D_{3}\right)$ is trivial, in agreement with Corollary 26. We give the list of generators and relations of $U_{n c}^{\gamma}$ for biquandles of cardinality 3 , with the corresponding $S_{0}$. 


\begin{tabular}{|c|c|c|c|c|}
\hline name & $\sigma$ & $\begin{array}{c}\text { generators } \\
\text { of } U_{n c}^{\gamma}\end{array}$ & equations & $S_{0}$ \\
\hline flip & $B Q_{1}^{3}$ & 6 & $f_{2} f_{1}=f_{1} f_{2}, f_{4} f_{3}=f_{3} f_{4}$, & - \\
$f_{6} f_{5}=f_{5} f_{6}$, & \\
\hline$a-f l i p\{2,3\} \cup\{1\}$ & $B Q_{2}^{3}$ & 3 & $f_{3} f_{2}=f_{2} f_{3}$, & - \\
\hline & $B Q_{3}^{3}$ & 3 & - & - \\
\hline & $B Q_{3}^{3 *}$ & 3 & - & - \\
\hline Wada $\left(\mathbb{Z}_{3}\right)$ & $B Q_{4}^{3}$ & 0 & - & $\{[1,2]\}$ \\
\hline inv. Wada $\left(\mathbb{Z}_{3}\right)$ & $B Q_{4}^{3 *}$ & 1 & $f_{1}^{3}=1$ & - \\
\hline & $B Q_{5}^{3}$ & 2 & - & $\{[1,3]\}$ \\
\hline$Q_{3}$ & $B Q_{6}^{3}$ & 2 & - & - \\
\hline inverse $Q_{3}$ & $B Q_{6}^{3 *}$ & 3 & - & $\{[1,2],[1,3]\}$ \\
\hline$(x, y) \mapsto(-y,-x)$ & $B Q_{7}^{3}$ & 3 & $f_{3} f_{2}=f_{2} f_{3}$, & - \\
\hline$D_{3}$ & $B Q_{8}^{3}$ & 0 & - & - \\
\hline inverse $D_{3}$ & $B Q_{8}^{3 *}$ & 0 & - & - \\
\hline & $B Q_{9}^{3}$ & 1 & $f_{1}^{3}=1$, & - \\
\hline involutive $\left(\mathbb{Z}_{3}\right)$ & $B Q_{9}^{3 *}$ & 0 & $f_{10} f_{2}=f_{2} f_{1}$ & - \\
\hline & & 2 & & - \\
\hline
\end{tabular}

We exhibit another example of computation using this algorithm:

\subsection{Reduced group for Alexander biquandle in $\mathbb{Z}_{4}$}

In order to choose a possible $\gamma$, we list as above elements of the form $\left(\bar{x},(x, y), \overline{\sigma^{2}(x, y)}\right)$ with $\left(\bar{x},(x, y) \neq \overline{\sigma^{2}(x, y)}\right)$. without repeating $\left(\bar{x},(x, y), \overline{\sigma^{2}(x, y)}\right)$, this gives only one element

$$
[0,[0,1], 2]]
$$

so we compute $U_{n c}^{\gamma}$ with $S_{0}=\{(0,1)\}$. The G.A.P. answer is

4 generators: $\left\{f_{1}=[2,1]=[4,1], f_{2}=[2,2]=[4,4], f_{3}=[2,3]=[4,3], f_{4}=[3,2]=[3,4]\right\}$, the trivial elements are

$$
1=[1,1]=[1,2]=[1,3]=[1,4]=[2,4]=[3,1]=[3,3]=[4,2]
$$

and relations

$$
\begin{gathered}
f_{1}=f_{2} f_{3}, f_{3}=f_{2} f_{1}, f_{1} f_{2}=f_{3}, f_{3} f_{2}=f_{1}, \\
f_{2} f_{1}=f_{1} f_{2}, f_{2} f_{3}=f_{3} f_{2}, f_{3} f_{1}=f_{1} f_{3}
\end{gathered}
$$

Notice that $f_{4}$ do not appear in the list of relations. Calling $a=f_{2}, b=f_{3}$, we get

$$
\begin{gathered}
f_{1}=a b, b=a f_{1}, f_{1} a=b, b a=f_{1} \\
b f_{1}=f_{1} b, a f_{1}=f_{1} a, a b=b a
\end{gathered}
$$

Replacing $f_{1}$ by $a b$ we get

$$
\begin{gathered}
b=a a b, a b a=b, b a=a b \\
b a b=a b b, a a b=a b a,
\end{gathered}
$$


whose solution is

$$
a^{2}=1, a b=b a
$$

so $U_{n c}^{\gamma}=\operatorname{Free}\left(a, b, f_{4}\right) /\left(a^{2}=1, a b=b a\right) \cong(\mathbb{Z} / 2 \mathbb{Z} \oplus \mathbb{Z}) * \mathbb{Z}$.

\subsection{Reduced Universal group of 4-cycles in $S_{4}$}

Another example of application of $U_{n c}^{\gamma}$ is the following: consider the quandle

$$
Q=\{(1,2,3,4),(1,2,4,3),(1,3,2,4),(1,3,4,2),(1,4,2,3),(1,4,3,2)\}
$$

that is, 4-cycles in $S_{4}$, with quandle operation $x \triangleleft y=y^{-1} x y$. Recall that $f: Q \times Q \rightarrow$ $U_{n c}(Q)$ is cohomologous to $f_{\gamma}$ if there exists a function $\gamma: Q \rightarrow U_{n c}$ such that

$$
f_{\gamma}(x, y)=\gamma(x) f(x, y) \gamma(x \triangleleft y)^{-1}
$$

If we list $\left(x,(x, y), \sigma^{2}(x, y)\right)$ without repeating "used" pairs $(x, x \triangleleft y)$, we get

$$
\begin{aligned}
& {[1,[1,2], 4],[1,[1,3], 6],[1,[1,4], 3],[1,[1,6], 2],[2,[2,1], 6],[2,[2,5], 4],} \\
& {[2,[2,6], 5],[3,[3,1], 4],[3,[3,4], 5],[3,[3,5], 6],[4,[4,2], 5],[5,[5,2], 6]}
\end{aligned}
$$

If we define $\gamma(1)=1, \gamma(4)=[1,2], \gamma(6)=[1,3], \gamma(3)=[1,4], \gamma(2)=[1,6], \gamma(5)=$ $\gamma(2)[2,6]$ then $S_{0}=\{[1,2],[1,3],[1,4],[1,6],[2,6]\}$. If we compute $U_{n c}$ using our algorithm, it gives 30 generators with with 108 equations, while $U_{n c}^{\gamma}$ has only 5 generators with 20 equations

$$
\begin{gathered}
1=f_{1} f_{3}, f_{2} f_{4}=1, f_{3} f_{1}=1, f_{5} f_{1}=1, f_{1} f_{5}=1, \\
f_{1} f_{1}=f_{2}, f_{1} f_{1}=f_{4}, f_{1} f_{1}=f_{3} f_{5}, f_{5}=f_{1} f_{4}, f_{1}=f_{2} f_{5}, \\
f_{1}=f_{3} f_{4}, f_{2} f_{1}=f_{3}, f_{4} f_{3}=f_{1}, f_{4} f_{1}=f_{5}, f_{5} f_{2}=f_{1}, \\
f_{1} f_{2}=f_{3}, f_{1} f_{2}=f_{2} f_{1}, f_{1} f_{3}=f_{3} f_{1}, f_{1} f_{4}=f_{4} f_{1}, f_{1} f_{5}=f_{5} f_{1},
\end{gathered}
$$

Call $a:=f_{1}$, then $f_{3}=f_{5}=a^{-1}, f_{2}=f_{4}=a^{2}$, and replacing these values into the 20 equations, the only remaining condition is $a^{4}=1$, we conclude $U_{n c}^{\gamma}=\left\langle a: a^{4}=1\right\rangle$

This quandle is interesting because it distinguish (using $U_{n c}^{\gamma}$ and its canonical cocycle) the trefoil from its mirror image: there are 30 colorings, 6 of them give trivial invariant both for the trefoil and its mirror (these are the 6 constant colorings), but the other 24 colorings gives $a^{-1}$ for the trefoil and $a$ for its mirror, and clearly $a \neq a^{-1}$ in $\left\langle a: a^{4}=1\right\rangle$.

\section{Some knots/links and their n.c. invariants}

There are 3 quandles of size 3, none of them give nontrivial invariant for knots up to 11 crossings. On the other hand, using the biquandle $B Q_{2}^{3}=$ aflip $\coprod\{1\}$, from the list of 84 knots with less or equal to 10 crossings, all of them have exactly 3 different colorings, but there are 44 with nontrivial invariant. For instance, figure eight has nontrivial invariant for tree biquandles of size 3: $B Q_{2}^{3}=$ aflip $\coprod\{1\}, B Q_{7}^{3}: \sigma(x, y)=(-y,-x)$, and $B Q_{9}^{3}$.

We illustrate in next table the number of colorings (denoted by $c$ ) and nontrivial invariants, for knots up to 6 crossings and biquandles from Bartholomew and Fenn's list: 


\begin{tabular}{c|c|cc|cc|c|c|c|cc} 
& $3_{1}$ & $4_{1}$ & & $5_{1}$ & & $5_{2}$ & $6_{1}$ & $6_{2}$ & $6_{3}$ & \\
& $c$ & $c$ & & $c$ & & $c$ & $c$ & $c$ & $c$ & \\
\hline$B Q_{1}^{3}$ & 3 & 3 & & 3 & & 3 & 3 & 3 & 3 & \\
$B Q_{2}^{3}$ & 3 & 3 & $f_{3}, f_{3}$ & 3 & $f_{3}^{-1}, f_{3}^{-1}$ & 3 & 3 & 3 & 3 & $f_{3}, f_{3}$, \\
\hline$B Q_{3}^{3}$ & 3 & 3 & & 3 & & 3 & 3 & 3 & 3 & \\
$B Q_{4}^{3}$ & 9 & 1 & & 1 & & 3 & 9 & 3 & 1 & \\
\hline$B Q_{5}^{3}$ & 3 & 3 & & 3 & & 3 & 3 & 3 & 3 & \\
$B Q_{6}^{3}$ & 3 & 3 & & 3 & & 3 & 3 & 3 & 3 & \\
\hline$B Q_{7}^{3}$ & 3 & 3 & $f_{3}, f_{3}$ & 3 & $f_{3}^{-1}, f_{3}^{-1}$ & 3 & 3 & 3 & 3 & $f_{3}, f_{3}$ \\
$B Q_{8}^{3}$ & 9 & 3 & & 3 & & 3 & 9 & 3 & 3 & \\
\hline$B Q_{9}^{3}$ & 9 & 3 & $f_{1}, f_{1}, f_{1}$ & 3 & $f_{1}^{-1}, f_{1}^{-1}, f_{1}^{-1}$ & 3 & 9 & 3 & 3 & $f_{1}, f_{1}, f_{1}$ \\
$B Q_{10}^{3}$ & 3 & 0 & & 0 & & 3 & 3 & 3 & 0 &
\end{tabular}

We see that using only number of colorings we can separate this list of knots in 3 groups: $\left\{3_{1}, 6_{1}\right\},\left\{4_{1}, 5_{1}, 6_{3}\right\}$ and $\left\{5_{2}, 6_{2}\right\}$, and using the invariant we can also distinguish $5_{1}$ from all others. One interesting remark is that, using only quandles, there are always the trivial constant colorings, but using biquandles it may happens that a knot admit no coloring at all, as we see with biquandle $B Q_{10}^{3}$.

\subsection{Wada inverse of $\mathbb{Z}_{3}$}

Recall for $\bar{\sigma}$ the inverse solution of Wada's for $G=\mathbb{Z}_{3}$, Unc $=\left\langle a: a^{3}=1\right\rangle$, the knot $7_{4}$ has nontrivial invariant under this biquandle solution (trivial invariant for one coloring, $a$ and $a^{2}$ for the other two). This example shows the importance of considering $\bar{\sigma}$ different from $\sigma$, since we have this example where for $\sigma=$ Wada's solution for $\mathbb{Z}_{3}$, every cocycle is coboundary and hence no invariant will appear, but for the inverse solution $\bar{\sigma}$ we get nontrivial things.

\subsection{Alexander biquandle on $\mathbb{Z}_{4}$ and $\mathbb{Z}_{8}$}

The Borromean link has trivial linking number, but has only 3 colorings using $D_{3}$, so we distinguish from three separated unknots. The Unc invariant are trivial for all biquandles of size 3 .

On the other hand, for the biAlexander biquandle on $\mathbb{Z}_{4}$ with $s=-1$ and $t=1$, even though there are 64 colorings, they give non trivial invariants:

Recall Unc $=$ Free $\left(a, b, f_{4}\right) /\left(b^{2}=1, a b=b a\right)$, the invariant for the Borromean link is trivial in 40 colorings, but gives twice $(\alpha, \alpha, 1),(\alpha, 1, \alpha),(1, \alpha, \alpha),\left(1, \alpha, \alpha^{-1}\right),\left(\alpha, 1, \alpha^{-1}\right)$, $\left(\alpha, \alpha^{-1}, 1\right)$ on the others, with $\alpha=a$ and $\alpha=a^{-1}$.,

In a similar way, Whitehead's link has trivial linking number, give trivial invariant for all biquandles of size 3 (even though non-trivial number of colorings), with bialexander on $\mathbb{Z}_{4}$ also give trivial invariant, but with with biAlexander on $\mathbb{Z}_{8}$ one has non trivial invariants. First we compute $U_{n c}^{\gamma}$ for $\mathbb{Z}_{8}, t=1, s=-1$, with subset $S_{0}=\{[1,2],[1,3],[2,2]\}$ (it may be seen that this is a subset corresponding to a convenient $\gamma$ ). The algorithm 
gives as answer that $U_{n c}^{\gamma}$ has 4 generators:

$$
\begin{aligned}
& f_{1}=(2,1)=(2,7)=(4,1)=(4,7)=(6,1)=(6,3)=(8,1)=(8,3), \\
& f_{2}=(2,3)=(2,5)=(4,3)=(4,5)=(6,5)=(6,7)=(8,5)=(8,7), \\
& f_{3}=(2,4)=(2,6)=(4,2)=(4,4)=(6,6)=(6,8)=(8,4)=(8,6), \\
& f_{4}=(3,2)=(3,4)=(3,6)=(3,8)=(7,2)=(7,4)=(7,6)=(7,8),
\end{aligned}
$$

Trivial elements are

$$
\begin{gathered}
1=[1,1]=[1,2]=[1,3]=[1,4]=[1,5]=[1,6]=[1,7]=[1,8]=[2,2]=[2,8]=[3,1] \\
=[3,3]=[3,5]=[3,7]=[4,6]=[4,8]=[5,1]=[5,2]=[5,3]=[5,4]=[5,5]=[5,6] \\
=[5,7]=[5,8]=[6,2]=[6,4]=[7,1]=[7,3]=[7,5]=[7,7]=[8,2]=[8,8]
\end{gathered}
$$

with relations

$$
\begin{gathered}
f_{1} f_{3}=f_{2}, f_{3} f_{1}=f_{2}, f_{3} f_{2}=f_{1}, f_{3} f_{3}=1 \\
f_{2} f_{1}=f_{1} f_{2}, f_{2} f_{2}=f_{1} f_{1}, f_{2} f_{3}=f_{1}, f_{3} f_{1}=f_{1} f_{3}, f_{3} f_{2}=f_{2} f_{3}
\end{gathered}
$$

Calling $a:=f_{1}, b:=f_{3}$, we get

$$
a b=f_{2}, f_{2} a=a f_{2}, f_{2} f_{2}=a a, f_{2} b=a, b a=f_{2}, b a=a b, b f_{2}=a, b f_{2}=f_{2} b, b b=1
$$

so $b^{2}=1$, and $f_{2}=a b=b a$. we conclude $U_{n c}^{\gamma}=\operatorname{Free}\left(a, b, f_{4}\right) /\left(b^{2}=1, a b=b a\right)$.

If we use this biquandle with Whitehead's link we get 64 colorings, 32 of them give trivial invariant, 16 colorings give $(b, 1)$ and 16 colorings give as invariant $(1, b)$.

\section{$5 \quad$ Final comments}

In the examples we saw, very often the group $U_{n c}^{\gamma}$ is non commutative, but we haven't found a knot/link with genuine non commutative invariant, that is, for example a commutator of two non commuting elements of $U_{n c}^{\gamma}$. Also, sometimes $U_{n c}^{\gamma}$ have pairs of commuting elements and other non commuting, for instance, $U_{n c}^{\gamma}\left(\operatorname{biAlex}\left(Z_{8}\right)\right)=\operatorname{Free}\left(a, b, f_{4}\right) /\left(b^{2}=\right.$ $1, a b=b a)$, but using this biquandle, computing the invariants for knots and links with less than 11 crossings, the elements $a$ and $b$ do not "mix" with $f_{4}$. We don't know if this is a general fact or not, that is, if the invariant obtained is the same if we use the abelianization of $U_{n c}^{\gamma}$.

If $U_{n c}^{\gamma}$ happens to be abelian, then the information we get with the non commutative invariant is essentially the state-sum invariant for the canonical cocycle $\pi_{\gamma}: X \times X \rightarrow U_{n c}^{\gamma}$. If this is the case (or if one consider the abelianization of $U_{n c}^{\gamma}$ ), then our construction can be seen as a natural and nontrivial way to produce interesting 2-cocycles, so that satesum invariant becomes a procedure with input only a biquandle, and not a biquandle plus a 2-cocycle, because a natural 2-cocycle is always present when one gives a biquandle.

Another natural question about state-sum invariant for biquandles is how to generalize it for 2-cocycles with values in nontrivial coefficients, which is known for quandles, but unknown for biquandles. In order to answer this question, it should be convenient to have an action of some group (to be defined) into the abelian group of coefficients where 
the 2-cocycle takes values, and if one imitates the quandle case, one should define, for each crossing, an exponent (in this group) that twist the value of the cocycle at that crossing. If the exponent is well-define, that is, for instance it remains unchanged under Reidemeister moves of other crossings, then essentially it must be a non commutative 2-cocycle. The group $U_{n c}$ was the candidate, and in fact this was origin of the present work. In the quandle case there is a natural map $U_{n c}(X) \rightarrow G_{X}$, where $G_{X}$ is the group generated by $X$ with relations $x y=z t$ if $\sigma(x, y)=(z, t)$; the map $U_{n c}(X) \rightarrow G_{X}$ is simply determined by $\left(x_{1}, x_{2}\right) \mapsto x_{2}$. So, for quandles, $G_{X}$-modules are natural candidates for coefficients (see [CEGS]), or also $U_{n c}(X)$-modules, or quandle-modules as considered in [AG]. We hope 2-cocycles with values in $U_{n c}$-modules will allow to define more general state-sum invariants, but at the moment we don't know how, we end remarking that for biquandles, there is no general well-defined map $U_{n c}(X) \rightarrow G_{X}$, and $U_{n c}(X)$ sometimes is the trivial group.

In [FG] one can found the GAP programs computing colorings, $U_{n c}^{\gamma}$, and invariants for knots and links given as planar diagrams.

\section{References}

[AG] N. Andruskiewitsch, M. Graña, From racks to pointed Hopf algebras. Adv. Math. 178 (2003), no. 2, 177-243.

[BF] A. Bartolomew, R. Fenn: Biquandles of Small Size and some Invariants of Virtual and Welded Knots, to appear. See also http://www.layer8.co.uk/maths/biquandles/index.htm

[CEGS] Carter, J.Scott; El Hamdadi, Mohamed; Graña, Matias; Saito, Masahico Cocycle knot invariants from quandle modules and generalized quandle homology. Zbl 1089.57008 Osaka J. Math. 42, No. 3, 499-541 (2005).

[CES] J.Scott Carter, Mohamed Elhamdadi and Masahico Saito, Homology Theory for the Set-Theoretic Yang-Baxter Equation and Knot Invariants from Generalizations of Quandles. Fund. Math. 184 (2004), 31-54.

[FG] M. Farinati - J. Garcia Galofre, http://mate.dm.uba.ar/ mfarinat/papers/GAP

[GAP2015] The GAP Group, GAP - Groups, Algorithms, and Programming, Version 4.7.8; 2015. http://www.gap-system.org

[G] M. Graña, Indecomposable racks of order $p^{2}$, Beiträge Algebra Geom. 45 2, 665-676 (2004). 